\title{
Completing the spectrum of almost resolvable cycle systems with odd cycle length
}

\author{
L. Wang and H. Cao * \\ Institute of Mathematics, \\ Nanjing Normal University, Nanjing 210023, China
}

\begin{abstract}
In this paper, we construct almost resolvable cycle systems of order $4 k+1$ for odd $k \geq 11$. This completes the proof of the existence of almost resolvable cycle systems with odd cycle length. As a by-product, some new solutions to the Hamilton-Waterloo problem are also obtained.
\end{abstract}

Key words: cycle system; almost resolvable cycle system; Hamilton-Waterloo problem

\section{Introduction}

In this paper, we use $V(H)$ and $E(H)$ to denote the vertex-set and the edge-set of a graph $H$, respectively. We denote the cycle of length $k$ by $C_{k}$ and the complete graph on $v$ vertices by $K_{v}$. A factor of a graph $H$ is a spanning subgraph whose vertex-set coincides with $V(H)$. If its connected components are isomorphic to $G$, we call it a $G$-factor. A $G$-factorization of $H$ is a set of edge-disjoint $G$-factors of $H$ whose edge-sets partition $E(H)$. A $C_{k}$-factorization of $H$ is a partition of $E(H)$ into $C_{k}$-factors. An $r$-regular factor is called an $r$-factor. Also, a 2-factorization of a graph $H$ is a partition of $E(H)$ into 2-factors.

A $k$-cycle system of order $v$ is a collection of $k$-cycles which partition $E\left(K_{v}\right)$. A $k$-cycle system of order $v$ exists if and only if $3 \leq k \leq v, v \equiv 1(\bmod 2)$ and $v(v-1) \equiv 0(\bmod 2 k)$ [2, 26]. A $k$-cycle system of order $v$ is resolvable if it has a $C_{k}$-factorization. A resolvable $k$-cycle system of order $v$ exists if and only if $3 \leq k \leq v, v$ and $k$ are odd, and $v \equiv 0(\bmod k)$, see [3, 4, 15, 19, 20, 24, 25]. If $v \equiv 1(\bmod 2 k)$, then a $k$-cycle system exists, but it is not resolvable. In this case, Vanstone et al. [27] started the research of the existence of an almost resolvable $k$-cycle system.

In a $k$-cycle system of order $v$, a collection of $(v-1) / k$ disjoint $k$-cycles is called an almost parallel class. In a $k$-cycle system of order $v \equiv 1(\bmod 2 k)$, the maximum possible number

${ }^{*}$ Research supported by the National Natural Science Foundation of China under Grant 11571179, and the Priority Academic Program Development of Jiangsu Higher Education Institutions. E-mail: caohaitao@njnu.edu.cn 
of almost parallel classes is $(v-1) / 2$, in which case a half-parallel class containing $(v-1) / 2 k$ disjoint $k$-cycles is left over. A $k$-cycle system of order $v$ whose cycle set can be partitioned into $(v-1) / 2$ almost parallel classes and a half-parallel class is called an almost resolvable $k$-cycle system, denoted by $k$-ARCS $(v)$.

For recursive constructions of almost resolvable $k$-cycle systems, C. C. Lindner, et al. [18] have considered the general existence problem of almost resolvable $k$-cycle system from the commutative quasigroup for $k \equiv 0(\bmod 2)$ and made a hypothesis: if there exists a $k$-ARCS $(2 k+1)$ for $k \equiv 0(\bmod 2)$ and $k \geq 8$, then there exists a $k$-ARCS $(2 k t+1)$ except possibly for $t=2$. H. Cao et al. [12, 22, 30] continued to consider the recursive constructions of an almost resolvable $k$-cycle system for $k \equiv 1(\bmod 2)$. Many authors contributed to the following known results.

Theorem 1.1. ([1, 6 6,12 14, 18, 27] $)$ Let $k \geq 3, t \geq 1$ be integers and $n=2 k t+1$. There exists a $k$-ARCS $(n)$ for $k \in\{3,4,5,6,7,8,9,10,14\}$, except for $(k, n) \in\{(3,7),(3,13),(4,9)\}$ and except possibly for $(k, n) \in\{(8,33),(14,57)\}$.

Theorem 1.2. $([22,[30])$ For any odd $k \geq 11$, there exists a $k$-ARCS $(2 k t+1)$, where $t \geq 1$ and $t \neq 2$.

In this paper, we construct almost resolvable cycle systems of order $4 k+1$ for odd $k \geq 11$. Combining the known results in Theorems 1.1]1.2, we will prove the following main result.

Theorem 1.3. For any odd $k \geq 3$, there exists a $k$-ARCS $(2 k t+1)$ for all $t \geq 1$ except for $(k, t) \in\{(3,1),(3,2)\}$.

\section{Preliminary}

In this section we present a basic lemma for the construction of a $k$-ARCS $(4 k+1)$. The main idea is to find some initial cycles with special properties such that all the required almost parallel classes can be obtained from them. We need the following notions for that lemma.

Suppose $\Gamma$ is an additive group and $I=\left\{\infty_{1}, \infty_{2}, \ldots, \infty_{f}\right\}$ is a set which is disjoint with $\Gamma$. We will consider an action of $\Gamma$ on $\Gamma \cup I$ which coincides with the right regular action on the elements of $\Gamma$, and the action of $\Gamma$ on $I$ will coincide with the identity map. In other words, for any $\gamma \in \Gamma$, we have that $x+\gamma$ is the image under $\gamma$ of any $x \in \Gamma$, and $x+\gamma=x$ holds for any $x \in I$. Given a graph $H$ with vertices in $\Gamma \cup I$, the translate of $H$ by an element $\gamma$ of $\Gamma$ is the graph $H+\gamma$ obtained from $H$ by replacing each vertex $x \in V(H)$ with the vertex $x+\gamma$. The development of $H$ under a subgroup $\Sigma$ of $\Gamma$ is the collection $\operatorname{dev}_{\Sigma}(H)=\{H+x \mid x \in \Sigma\}$ of all translates of $H$ by an element of $\Sigma$.

For our constructions, we set $\Gamma=\mathbb{Z}_{k} \times \mathbb{Z}_{4}$. Given a graph $H$ with vertices in $\Gamma$ and any pair $(r, s) \in \mathbb{Z}_{4} \times \mathbb{Z}_{4}$, we set $\Delta_{(r, s)} H=\{x-y \mid\{(x, r),(y, s)\} \in E(H)\}$. Finally, given a list 
$\mathcal{H}=\left\{H_{1}, H_{2}, \ldots, H_{t}\right\}$ of graphs, we denote by $\Delta_{(r, s)} \mathcal{H}=\cup_{i=1}^{t} \Delta_{(r, s)} H_{i}$ the multiset union of the $\Delta_{(r, s)} H_{i} \mathrm{~s}$.

Lemma 2.1. Let $v=4 k+1$ and $\mathcal{C}=\left\{F_{1}, F_{2}\right\}$ where each $F_{i}(i=1,2)$ is a vertex-disjoint union of four cycles of length $k$ satisfying the following conditions:

(i) $V\left(F_{i}\right)=\left(\left(\mathbb{Z}_{k} \times \mathbb{Z}_{4}\right) \cup\{\infty\}\right) \backslash\left\{\left(a_{i}, b_{i}\right)\right\}$ for some $\left(a_{i}, b_{i}\right) \in \mathbb{Z}_{k} \times \mathbb{Z}_{4}, i=1,2$;

(ii) $\infty$ has a neighbor in $\mathbb{Z}_{k} \times\{j\}$ for each $j \in \mathbb{Z}_{4}$;

(iii) $\Delta_{(p, p)} \mathcal{C}=\mathbb{Z}_{k} \backslash\{0\}$ for each $p \in\{0,1\}$;

(iv) $\Delta_{(q, q)} \mathcal{C}=\mathbb{Z}_{k} \backslash\left\{0, \pm d_{q}\right\}$ for each $q \in\{2,3\}$, where $d_{q}$ satisfies $\left(d_{q}, k\right)=1$;

(v) $\Delta_{(r, s)} \mathcal{C}=\mathbb{Z}_{k}$ for each pair $(r, s) \in \mathbb{Z}_{4} \times \mathbb{Z}_{4}$ satisfying $r \neq s$.

Then, there exists a $k$-ARCS $(v)$.

Proof: Let $V\left(K_{v}\right)=\left(\mathbb{Z}_{k} \times \mathbb{Z}_{4}\right) \cup\{\infty\}$. Note that $0, d_{q}, 2 d_{q}, \ldots,(k-1) d_{q}$ are $k$ distinct elements since $\left(d_{q}, k\right)=1$. Then we have the required half parallel class which is formed by the two cycles $\left((0, q),\left(d_{q}, q\right),\left(2 d_{q}, q\right), \ldots,\left((k-1) d_{q}, q\right)\right), q=2,3$. By $(i)$, we know that $F_{i}$ is an almost parallel class. All the required $2 k$ almost parallel classes are $F_{i}+(l, 0), i=1,2$, $l \in \mathbb{Z}_{k}$.

Now we show that the half parallel class and the $2 k$ almost parallel classes form a $k$ $\operatorname{ARCS}(v)$. Let $F^{\prime}$ be a graph with the edge-set $\left\{\left\{(a, q),\left(a+d_{q}, q\right)\right\} \mid a \in \mathbb{Z}_{k}, q=2,3\right\}$ and $\Sigma:=\mathbb{Z}_{k} \times\{0\}$. Let $\mathcal{F}=\operatorname{dev}_{\Sigma}(\mathcal{C}) \cup F^{\prime}$. The total number of edges - counted with their respective multiplicities - covered by the almost parallel classes and the half parallel class of $\mathcal{F}$ is $2 k(4 k+1)$, that is exactly the size of $E\left(K_{v}\right)$. Therefore, we only need to prove that every pair of vertices lies in a suitable translate of $\mathcal{C}$ or in $F^{\prime}$. By $(i i)$, an edge $\{(z, j), \infty\}$ of $K_{v}$ must appear in a cycle of $\operatorname{dev}_{\Sigma}(\mathcal{C})$.

Now consider an edge $\left\{(z, j),\left(z^{\prime}, j^{\prime}\right)\right\}$ of $K_{v}$ whose vertices both belong to $\mathbb{Z}_{k} \times \mathbb{Z}_{4}$. If $j=j^{\prime} \in\{2,3\}$ and $z-z^{\prime} \in\left\{ \pm d_{q}\right\}$, then this edge belongs to $F^{\prime}$. In all other cases there is, by $(i i i)-(v)$, an edge of some $F_{i}$ of the form $\left\{(w, j),\left(w^{\prime}, j^{\prime}\right)\right\}$ such that $w-w^{\prime}=z-z^{\prime}$. It then follows that $F_{i}+\left(-w^{\prime}+z^{\prime}, 0\right)$ is an almost parallel class of $\operatorname{dev}_{\Sigma}\left(F_{i}\right)$ containing the edge $\left\{(z, j),\left(z^{\prime}, j^{\prime}\right)\right\}$ and the conclusion follows.

\section{$3 \quad k$-ARCS $(4 k+1)$ for $k \equiv 1(\bmod 4)$}

In this section, we will prove the existence of a $k$-ARCS $(4 k+1)$ for $k \equiv 1(\bmod 4)$.

Lemma 3.1. For any $k \geq 13$ and $k \equiv 1(\bmod 4)$, there exists a $k$-ARCS $(4 k+1)$.

Proof: Let $v=4 k+1$ and $k=4 n+1, n \geq 3$. We use Lemma 2.1 to construct a $k$-ARCS $(v)$ with $V\left(K_{v}\right)=\left(\mathbb{Z}_{k} \times \mathbb{Z}_{4}\right) \cup\{\infty\}$. The required parameters in $(i)$ and (iv) of Lemma 2.1 are $\left(a_{1}, b_{1}\right)=(0,3),\left(a_{2}, b_{2}\right)=(0,2), d_{2}=2$, and $d_{3}=\frac{k-1}{2}$. The required 8 cycles in $F_{1}=\left\{C_{1}, C_{2}, C_{3}, C_{4}\right\}$ and $F_{2}=\left\{C_{5}, C_{6}, C_{7}, C_{8}\right\}$ are listed as below. 
The cycle $C_{1}$ is the concatenation of the sequences $T_{1},(0,0)$, and $T_{2}$, where $T_{1}=((n, 0),(-n, 1), \ldots, \underline{(n-i, 0),(-(n-i), 1)}, \ldots,(1,0),(-1,1)), 0 \leq i \leq n-1 ;$ $T_{2}=((1,1),(-1,0), \ldots, \underline{(1+i, 1),(-(1+i), 0)}, \ldots,(n, 1),(-n, 0)), 0 \leq i \leq n-1$.

Note: Actually $T_{1}$ can be viewed as the concatenation of the sequences $T_{1}^{0}, T_{1}^{1}, \ldots, T_{1}^{n-1}$, where the general formula is $T_{1}^{i}=((n-i, 0),(-(n-i), 1)), 0 \leq i \leq n-1$. Thus, for brevity, we just list the first sequence at the beginning of $T_{1}$ and the last sequence at the end of $T_{1}$, and use the underlined sections to give the general formula in the middle of $T_{1}$. We give the range of $i$ after the sequence $T_{1}$ so that the reader can easily calculate the number of vertices in $T_{1}$. Similarly, this partial underlining happens ahead in some places as well.

The cycle $C_{2}$ is the concatenation of the sequences $T_{1}, T_{2}$, and $(0,2)$, where $T_{1}=((1,2),(-1,3), \ldots, \underline{(1+i, 2),(-(1+i), 3)}, \ldots,(n, 2),(-n, 3)), 0 \leq i \leq n-1$; $T_{2}=((-n, 2),(n, 3), \ldots,(-(n-i), 2),(n-i, 3), \ldots,(-1,2),(1,3)), 0 \leq i \leq n-1$.

The cycle $C_{3}$ is the concatenation of the sequences $T_{1}, T_{2}$, and $T_{3}$, where $T_{1}=((n+1,1),(-(n+1), 2), \ldots,(n+1+i, 1),(-(n+1+i), 2), \ldots,(2 n-1,1),(-(2 n-1), 2)), 0 \leq i \leq n-2 ;$ $T_{2}=((-2 n, 1),(2 n, 2), \ldots, \underline{(-(2 n-i), 1),(2 n-i, 2)}, \ldots,(-(n+1), 1),(n+1,2)), 0 \leq i \leq n-1$; $T_{3}=((-2 n, 0),(-2 n, 2),(0,1))$.

The cycle $C_{4}$ is the concatenation of the sequences $\infty, T_{1}, T_{2}$, and $T_{3}$ (By a slight abuse of notation, here $\infty$ is regarded as a sequence.), where $T_{1}=((-2 n, 3),(2 n, 3), \ldots, \underline{(-(2 n-i), 3),(2 n-i, 3)}, \ldots,(-(n+1), 3),(n+1,3)), 0 \leq i \leq n-1 ;$ $T_{2}=((n+1,0),(-(n+1), 0), \ldots, \underline{(n+1+i, 0),(-(n+1+i), 0)}, \ldots,(2 n-1,0),(-(2 n-1), 0)), 0 \leq i \leq n-2 ;$ $T_{3}=((2 n, 0),(2 n, 1))$.

The cycle $C_{5}$ is the concatenation of the sequences $T_{1},(0,1)$, and $T_{2}$, where $T_{1}=((n, 1),(-n, 3), \ldots, \underline{(n-i, 1),(-(n-i), 3)}, \ldots,(1,1),(-1,3)), 0 \leq i \leq n-1 ;$ $T_{2}=((1,3),(-1,1), \ldots, \underline{(1+i, 3),(-(1+i), 1)}, \ldots,(n, 3),(-n, 1)), 0 \leq i \leq n-1$.

The cycle $C_{6}$ is the concatenation of the sequences $T_{1}, T_{2}, T_{3}$, and $(-n, 0)$, where $T_{1}=((-(n+1), 0),(n+1,3), \ldots, \underline{(-(n+1+i), 0),(n+1+i, 3)}, \ldots,(-(2 n-1), 0),(2 n-1,3)), 0 \leq i \leq n-2 ;$ $T_{2}=((-2 n, 0),(0,3))$; $T_{3}=((2 n, 0),(-2 n, 3), \ldots, \underline{(2 n-i, 0),(-(2 n-i), 3)}, \ldots,(n+1,0),(-(n+1), 3)), 0 \leq i \leq n-1$.

Next, we consider the cycles $C_{7}$ and $C_{8}$.

For $k=13,25$, the two cycles are listed as follows. $k=13:$

$C_{7}=((1,2),(-5,1),(-5,2),(0,0),(3,2),(-2,0),(4,2),(3,0),(5,2),(1,0),(-3,2),(-1,0),(-2,2)) ;$ $C_{8}=(\infty,(2,0),(-4,2),(-6,1),(5,1),(4,1),(-4,1),(6,1),(6,3),(-6,2),(2,2),(6,2),(-1,2))$. $k=25$ : $C_{7}=((1,2),(-11,1),(-11,2),(0,0),(2,2),(1,0),(4,2),(5,0),(3,2),(6,0),(10,2),(2,0),(7,2),(-5,0),(5,2)$, $(-4,0),(11,2),(4,0),(-8,2),(-1,0),(-10,2),(-2,0),(-7,2),(-3,0),(8,2))$; $C_{8}=(\infty,(3,0),(9,2),(7,1),(-7,1),(8,1),(-8,1),(9,1),(11,1),(-10,1),(10,1),(-9,1),(-12,1),(12,1)$, 
$(12,3),(-12,2),(-6,2),(6,2),(-3,2),(12,2),(-2,2),(-5,2),(-1,2),(-9,2),(-4,2))$.

For $k \geq 17$ and $k \neq 25$, we distinguish the following two cases.

Case $1: k \equiv 5(\bmod 8)$ and $k \geq 21$.

In this case, $C_{7}$ is the concatenation of the sequences $S_{1}, S_{2}, \ldots, S_{6}$ as follows.

$S_{1}=((-(2 n-3), 2),(-(2 n-1), 1))$;

$S_{2}=\left((1,2),(-1,0), \ldots, \underline{(1+i, 2),(-(1+i), 0)}, \ldots,\left(\frac{n-1}{2}, 2\right),\left(-\frac{n-1}{2}, 0\right)\right), 0 \leq i \leq \frac{n-3}{2}$;

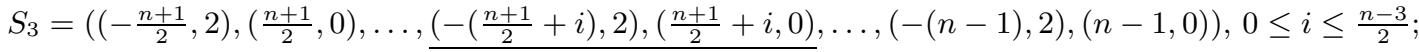

$S_{4}=((2 n-1,2),(0,0),(-(2 n-1), 2))$;

$S_{5}=\left((1,0),(-1,2), \ldots, \underline{(1+i, 0),(-(1+i), 2)}, \ldots,\left(\frac{n-1}{2}, 0\right),\left(-\frac{n-1}{2}, 2\right)\right), 0 \leq i \leq \frac{n-3}{2} ;$

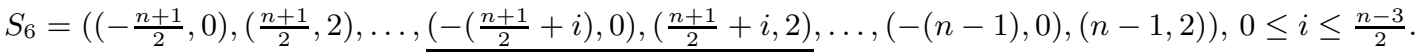

For the last cycle $C_{8}$, when $k=21,29$, it is listed as below respectively.

$k=21$ :

$C_{8}=(\infty,(5,0),(-6,2),(-6,1),(6,1),(-7,1),(7,1),(9,1),(-8,1),(8,1),(-10,1),(10,1),(10,3),(-10,2)$,

$(8,2),(-5,2),(7,2),(-8,2),(6,2),(10,2),(5,2))$.

$k=29$ :

$C_{8}=(\infty,(7,0),(-8,2),(-8,1),(8,1),(-9,1),(9,1),(-10,1),(10,1),(-14,1),(-12,1),(13,1),(12,1),(-11,1)$,

$(11,1),(14,1),(14,3),(-14,2),(7,2),(11,2),(14,2),(8,2),(-7,2),(9,2),(-9,2),(10,2),(-10,2),(12,2)$,

$(-12,2))$.

When $k \geq 37, C_{8}$ is the concatenation of the sequences $\infty, T_{1}, T_{2}, \ldots, T_{8}$, where $T_{1}=((n, 0),(-(n+1), 2))$;

$T_{2}=\left((-(n+1), 1),(n+1,1), \ldots, \underline{(-(n+1+i), 1),(n+1+i, 1)}, \ldots,\left(-\frac{3 n-1}{2}, 1\right),\left(\frac{3 n-1}{2}, 1\right)\right), 0 \leq i \leq \frac{n-3}{2}$.

For the other 6 sequences $T_{3}, T_{4}, \ldots, T_{8}$, we distinguish the following 3 subcases.

Case 1.1: $k \equiv 5(\bmod 24)$ and $k \geq 53$.

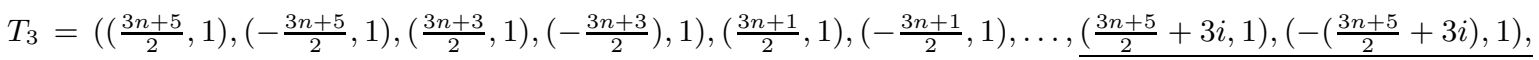

$\left(\frac{3 n+3}{2}+3 i, 1\right),\left(-\left(\frac{3 n+3}{2}+3 i\right), 1\right),\left(\frac{3 n+1}{2}+3 i, 1\right),\left(-\left(\frac{3 n+1}{2}+3 i\right), 1\right), \ldots,(2 n-7,1),(-(2 n-7), 1)$,

$(2 n-8,1),(-(2 n-8), 1),(2 n-9,1),(-(2 n-9), 1)), 0 \leq i \leq \frac{n-19}{6}$;

$T_{4}=((2 n-4,1),(-(2 n-3), 1),(2 n-3,1),(-(2 n-2), 1),(2 n-2,1),(-(2 n-6), 1),(2 n-6,1),(-(2 n-5), 1)$,

$(2 n-5,1),(-(2 n-4), 1),(-2 n, 1),(2 n-1,1),(2 n, 1),(2 n, 3),(-2 n, 2),(n, 2),(-n, 2))$;

$T_{5}=((n+2,2),(-(n+2), 2),(n+1,2),(-(n+4), 2),(n+3,2),(-(n+3), 2), \ldots,(n+2+3 i, 2),(-(n+2+3 i), 2)$,

$\underline{(n+1+3 i, 2),(-(n+4+3 i), 2),(n+3+3 i, 2),(-(n+3+3 i), 2)}, \ldots,\left(\frac{3 n-9}{2}, 2\right),\left(-\frac{3 n-9}{2}, 2\right),\left(\frac{3 n-11}{2}, 2\right)$,

$\left.\left(-\frac{3 n-5}{2}, 2\right),\left(\frac{3 n-7}{2}, 2\right),\left(-\frac{3 n-7}{2}, 2\right)\right), 0 \leq i \leq \frac{n-13}{6}$;

$T_{6}=\left(\left(\frac{3 n-3}{2}, 2\right),\left(-\frac{3 n-3}{2}, 2\right),\left(\frac{3 n-1}{2}, 2\right),\left(-\frac{3 n-1}{2}, 2\right),\left(\frac{3 n+3}{2}, 2\right),\left(\frac{3 n-5}{2}, 2\right)\right) ;$

$T_{7}=\left(\left(\frac{3 n+5}{2}, 2\right),\left(-\frac{3 n+3}{2}, 2\right),\left(\frac{3 n+1}{2}, 2\right),\left(-\frac{3 n+5}{2}, 2\right),\left(\frac{3 n+9}{2}, 2\right),\left(-\frac{3 n+1}{2}, 2\right), \ldots,\left(\frac{3 n+5}{2}+3 i, 2\right),\left(-\left(\frac{3 n+3}{2}+3 i\right), 2\right)\right.$,

$\left(\frac{3 n+1}{2}+3 i, 2\right),\left(-\left(\frac{3 n+5}{2}+3 i\right), 2\right),\left(\frac{3 n+9}{2}+3 i, 2\right),\left(-\left(\frac{3 n+1}{2}+3 i\right), 2\right), \ldots,(2 n-7,2),(-(2 n-8), 2)$,

$(2 n-9,2),(-(2 n-7), 2),(2 n-5,2),(-(2 n-9), 2)), 0 \leq i \leq \frac{n-19}{6}$;

$T_{8}=((2 n-4,2),(-(2 n-2), 2),(2 n, 2),(-(2 n-5), 2),(2 n-6,2),(-(2 n-4), 2),(2 n-3,2),(-(2 n-6), 2)$,

$(2 n-2,2))$. 
Case 1.2: $k \equiv 13(\bmod 24)$ and $k \geq 37$.

$$
\begin{aligned}
& T_{3}=\left(\left(\frac{3 n+5}{2}, 1\right),\left(-\frac{3 n+5}{2}, 1\right),\left(\frac{3 n+3}{2}, 1\right),\left(-\frac{3 n+3}{2}, 1\right),\left(\frac{3 n+1}{2}, 1\right),\left(-\frac{3 n+1}{2}, 1\right), \ldots, \frac{\left(\frac{3 n+5}{2}+3 i, 1\right),\left(-\left(\frac{3 n+5}{2}+3 i\right), 1\right),}{}\right. \\
& \frac{\left(\frac{3 n+3}{2}+3 i, 1\right),\left(-\left(\frac{3 n+3}{2}+3 i\right), 1\right),\left(\frac{3 n+1}{2}+3 i, 1\right),\left(-\left(\frac{3 n+1}{2}+3 i\right), 1\right), \ldots,(2 n-2,1),(-(2 n-2), 1),}{(2 n-3,1),(-(2 n-3), 1),(2 n-4,1),(-(2 n-4), 1)), 0 \leq i \leq \frac{n-9}{6} ;} \\
& T_{4}=((-2 n, 1),(2 n-1,1),(2 n, 1),(2 n, 3),(-2 n, 2),(n, 2),(-n, 2)) ; \\
& T_{5}=\left((n+2,2),(-(n+2), 2),(n+1,2),(-(n+4), 2),(n+3,2),(-(n+3), 2), \ldots, \frac{(n+2+3 i, 2),(-(n+2+3 i), 2),}{}\right. \\
& \frac{(n+1+3 i, 2),(-(n+4+3 i), 2),(n+3+3 i, 2),(-(n+3+3 i), 2), \ldots,\left(\frac{3 n-11}{2}, 2\right),\left(-\frac{3 n-11}{2}, 2\right),}{\left.\left(\frac{3 n-13}{2}, 2\right),\left(-\frac{3 n-7}{2}, 2\right),\left(\frac{3 n-9}{2}, 2\right),\left(-\frac{3 n-9}{2}, 2\right)\right), 0 \leq i \leq \frac{n-15}{6} ;} \\
& T_{6}=\left(\left(\frac{3 n-5}{2}, 2\right),\left(-\frac{3 n-5}{2}, 2\right),\left(\frac{3 n-7}{2}, 2\right),\left(\frac{3 n+5}{2}, 2\right),\left(\frac{3 n-3}{2}, 2\right),\left(-\frac{3 n-3}{2}, 2\right),\left(\frac{3 n-1}{2}, 2\right)\right) ; \\
& T_{7}=\left(\left(-\frac{3 n-1}{2}, 2\right),\left(\frac{3 n+11}{2}, 2\right),\left(-\frac{3 n+1}{2}, 2\right),\left(\frac{3 n+1}{2}, 2\right),\left(-\frac{3 n+3}{2}, 2\right),\left(\frac{3 n+3}{2}, 2\right), \ldots,\left(-\left(\frac{3 n-1}{2}+3 i\right), 2\right),\left(\frac{3 n+11}{2}+3 i, 2\right),\right. \\
& \frac{\left(-\left(\frac{3 n+1}{2}+3 i\right), 2\right),\left(\frac{3 n+1}{2}+3 i, 2\right),\left(-\left(\frac{3 n+3}{2}+3 i\right), 2\right),\left(\frac{3 n+3}{2}+3 i, 2\right), \ldots,(-(2 n-8), 2),(2 n-2,2),}{(-(2 n-7), 2),(2 n-7,2),(-(2 n-6), 2)),(2 n-6,2), 0 \leq i \leq \frac{n-15}{6} ;} \\
& T_{8}=((-(2 n-5), 2),(2 n-3,2),(-(2 n-4), 2),(2 n, 2),(-(2 n-2), 2),(2 n-4,2)) .
\end{aligned}
$$

Case 1.3: $k \equiv 21(\bmod 24)$ and $k \geq 45$.

$$
\begin{aligned}
& T_{3}=\left(\left(\frac{3 n+5}{2}, 1\right),\left(-\frac{3 n+5}{2}, 1\right),\left(\frac{3 n+3}{2}, 1\right),\left(-\frac{3 n+3}{2}, 1\right),\left(\frac{3 n+1}{2}, 1\right),\left(-\frac{3 n+1}{2}, 1\right), \ldots, \underline{\left(\frac{3 n+5}{2}+3 i, 1\right),\left(-\left(\frac{3 n+5}{2}+3 i\right), 1\right),}\right.
\end{aligned}
$$

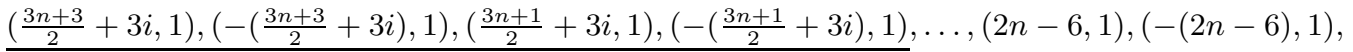

$$
\begin{aligned}
& (2 n-7,1),(-(2 n-7), 1),(2 n-8,1),(-(2 n-8), 1)), 0 \leq i \leq \frac{n-17}{6} \\
& T_{4}=((2 n-3,1),(-(2 n-3), 1),(2 n-2,1),(-(2 n-2), 1),(2 n-5,1),(-(2 n-5), 1),(2 n-4,1),(-(2 n-4), 1), \\
& (-2 n, 1),(2 n-1,1),(2 n, 1),(2 n, 3),(-2 n, 2),(n, 2),(-n, 2)) \text {; } \\
& T_{5}=((n+2,2),(-(n+2), 2),(n+1,2),(-(n+4), 2),(n+3,2),(-(n+3), 2), \ldots, \underline{(n+2+3 i, 2),(-(n+2+3 i), 2),} \\
& (n+1+3 i, 2),(-(n+4+3 i), 2),(n+3+3 i, 2),(-(n+3+3 i), 2), \ldots,\left(\frac{3 n-13}{2}, 2\right),\left(-\frac{3 n-13}{2}, 2\right), \\
& \left.\left(\frac{3 n-15}{2}, 2\right),\left(-\frac{3 n-9}{2}, 2\right),\left(\frac{3 n-11}{2}, 2\right),\left(-\frac{3 n-11}{2}, 2\right)\right), 0 \leq i \leq \frac{n-17}{6} ; \\
& T_{6}=\left(\left(\frac{3 n-7}{2}, 2\right),\left(-\frac{3 n-7}{2}, 2\right),\left(\frac{3 n-9}{2}, 2\right),\left(-\frac{3 n-3}{2}, 2\right),\left(\frac{3 n-1}{2}, 2\right),\left(-\frac{3 n-5}{2}, 2\right),\left(\frac{3 n-5}{2}, 2\right),\left(\frac{3 n+3}{2}, 2\right),\left(\frac{3 n-3}{2}, 2\right),\right. \\
& \left.\left(-\frac{3 n+1}{2}, 2\right),\left(\frac{3 n+1}{2}, 2\right)\right) \text {; } \\
& T_{7}=\left(\left(-\frac{3 n+3}{2}, 2\right),\left(\frac{3 n+7}{2}, 2\right),\left(-\frac{3 n-1}{2}, 2\right),\left(\frac{3 n+9}{2}, 2\right),\left(-\frac{3 n+7}{2}, 2\right),\left(\frac{3 n+5}{2}, 2\right), \ldots, \underline{\left(-\left(\frac{3 n+3}{2}+3 i\right), 2\right),\left(\frac{3 n+7}{2}+3 i, 2\right),}\right. \\
& \left(-\left(\frac{3 n-1}{2}+3 i\right), 2\right),\left(\frac{3 n+9}{2}+3 i, 2\right),\left(-\left(\frac{3 n+7}{2}+3 i\right), 2\right),\left(\frac{3 n+5}{2}+3 i, 2\right), \ldots,(-(2 n-7), 2),(2 n-5,2), \\
& (-(2 n-9), 2),(2 n-4,2),(-(2 n-5), 2),(2 n-6,2)), 0 \leq i \leq \frac{n-17}{6} ; \\
& T_{8}=((-(2 n-4), 2),(2 n-3,2),(-(2 n-2), 2),(2 n-2,2),(-(2 n-6), 2),(2 n, 2)) \text {. }
\end{aligned}
$$

Case $2: k \equiv 1(\bmod 8), k \geq 17$ and $k \neq 25$.

In this case, $C_{7}$ is the concatenation of the sequences $S_{1}, S_{2},(-n, 2), S_{3}, S_{4}, S_{5}$, and $S_{6}$. $S_{1}=((-(2 n-3), 2),(-(2 n-1), 1))$;

$S_{2}=((1,2),(-1,0), \ldots, \underline{(1+i, 2),(-(1+i), 0)}, \ldots,(n-1,2),(-(n-1), 0)), 0 \leq i \leq n-2$;

$S_{3}=\left((1,0),(-1,2), \ldots,(1+i, 0),(-(1+i), 2), \ldots,\left(\frac{n-2}{2}, 0\right),\left(-\frac{n-2}{2}, 2\right)\right), 0 \leq i \leq \frac{n-4}{2} ;$

$S_{4}=\left(\left(\frac{n}{2}, 0\right),\left(-\frac{3 n}{2}, 2\right)\right)$

$S_{5}=\left(\left(\frac{n+2}{2}, 0\right),\left(-\frac{n+2}{2}, 2\right), \ldots,\left(\frac{n+2}{2}+i, 0\right),\left(-\left(\frac{n+2}{2}+i\right), 2\right), \ldots,(n-1,0),(-(n-1), 2)\right), 0 \leq i \leq \frac{n-4}{2}$;

$S_{6}=((n, 0),(n+1,2))$.

For the cycle $C_{8}$, when $k=17,33,41$, it is listed as below respectively. 


$$
\begin{aligned}
& k=17 \text { : } \\
& C_{8}=(\infty,(0,0),(7,2),(7,1),(6,1),(-8,1),(-6,1),(5,1),(-5,1),(8,1),(8,3),(-8,2),(6,2),(-2,2),(-7,2) \text {, } \\
& (4,2),(8,2)) \text {. } \\
& k=33 \text { : } \\
& C_{8}=(\infty,(0,0),(15,2),(15,1),(9,1),(-9,1),(10,1),(-10,1),(11,1),(-11,1),(12,1),(13,1),(-12,1),(14,1) \text {, } \\
& (-14,1),(-16,1),(-13,1),(16,1),(16,3),(-16,2),(8,2),(12,2),(-14,2),(16,2),(-11,2),(14,2) \text {, } \\
& (-9,2),(10,2),(-10,2),(11,2),(-4,2),(13,2),(-15,2)) \text {. } \\
& k=41 \text { : } \\
& C_{8}=(\infty,(0,0),(19,2),(19,1),(-12,1),(13,1),(-13,1),(11,1),(-11,1),(12,1),(-15,1),(14,1),(-14,1) \text {, } \\
& (18,1),(15,1),(16,1),(-17,1),(17,1),(-18,1),(-20,1),(-16,1),(20,1),(20,3),(-20,2),(10,2), \\
& (13,2),(-13,2),(14,2),(-11,2),(12,2),(-12,2),(17,2),(-5,2),(16,2),(-19,2),(18,2),(-14,2) \text {, } \\
& (20,2),(-16,2),(15,2),(-18,2)) \text {. }
\end{aligned}
$$

When $k \geq 49$, the cycle $C_{8}$ is the concatenation of the sequences $\infty, T_{1}, T_{2}, \ldots, T_{9}$, where $T_{1}=((0,0),(2 n-1,2),(2 n-1,1))$.

For the other 8 sequences $T_{2}, T_{3}, \ldots, T_{9}$, we distinguish 3 subcases.

Case 2.1: $k \equiv 1(\bmod 24)$ and $k \geq 49$.

$$
\begin{aligned}
& T_{2}=((-(n+2), 1),(n+3,1),(-(n+3), 1),(n+1,1),(-(n+1), 1),(n+2,1), \ldots, \underline{(-(n+2+3 i), 1),(n+3+3 i, 1)}, \\
& \underline{(-(n+3+3 i), 1),(n+1+3 i, 1),(-(n+1+3 i), 1),(n+2+3 i, 1)}, \ldots,\left(-\frac{3 n-8}{2}, 1\right),\left(\frac{3 n-6}{2}, 1\right),\left(-\frac{3 n-6}{2}, 1\right), \\
& \left.\left(\frac{3 n-10}{2}, 1\right),\left(-\frac{3 n-10}{2}, 1\right),\left(\frac{3 n-8}{2}, 1\right)\right), 0 \leq i \leq \frac{n-12}{6} \text {; } \\
& T_{3}=\left(\left(-\frac{3 n-2}{2}, 1\right),\left(\frac{3 n}{2}, 1\right),\left(\frac{3 n-2}{2}, 1\right),\left(-\frac{3 n-4}{2}, 1\right),\left(\frac{3 n-4}{2}, 1\right),\left(-\frac{3 n}{2}, 1\right),\left(\frac{3 n+4}{2}, 1\right),\left(-\frac{3 n+2}{2}, 1\right),\left(-\frac{3 n+6}{2}, 1\right),\left(\frac{3 n+2}{2}, 1\right)\right) \text {; } \\
& T_{4}=\left(\left(-\frac{3 n+8}{2}, 1\right),\left(\frac{3 n+8}{2}, 1\right),\left(-\frac{3 n+4}{2}, 1\right),\left(\frac{3 n+10}{2}, 1\right),\left(-\frac{3 n+12}{2}, 1\right),\left(\frac{3 n+6}{2}, 1\right), \ldots, \underline{\left(-\left(\frac{3 n+8}{2}+3 i\right), 1\right),\left(\frac{3 n+8}{2}+3 i, 1\right),}\right. \\
& \frac{\left(-\left(\frac{3 n+4}{2}+3 i\right), 1\right),\left(\frac{3 n+10}{2}+3 i, 1\right),\left(-\left(\frac{3 n+12}{2}+3 i\right), 1\right),\left(\frac{3 n+6}{2}+3 i, 1\right)}{(-(2 n-7), 1),(2 n-4,1),(-(2 n-3), 1),(2 n-6,1)), 0 \leq i \leq \frac{n-18}{6}} ;,(-(2 n-5), 1),(2 n-5,1), \\
& T_{5}=((-(2 n-2), 1),(2 n-3,1),(-2 n, 1),(2 n-2,1),(-(2 n-4), 1),(2 n, 1),(2 n, 3),(-2 n, 2),(n, 2)) \text {; } \\
& T_{6}=((n+4,2),(-(n+3), 2),(n+3,2),(-(n+2), 2),(n+2,2),(-(n+1), 2), \ldots, \underline{(n+4+3 i, 2),(-(n+3+3 i), 2),} \\
& (n+3+3 i, 2),(-(n+2+3 i), 2),(n+2+3 i, 2),(-(n+1+3 i), 2), \ldots,\left(\frac{3 n-4}{2}, 2\right),\left(-\frac{3 n-6}{2}, 2\right),\left(\frac{3 n-6}{2}, 2\right), \\
& \left.\left(-\frac{3 n-8}{2}, 2\right),\left(\frac{3 n-8}{2}, 2\right),\left(-\frac{3 n-10}{2}, 2\right)\right), 0 \leq i \leq \frac{n-12}{6} \text {; } \\
& T_{7}=\left(\left(\frac{3 n+2}{2}, 2\right),\left(-\frac{n}{2}, 2\right),\left(\frac{3 n-2}{2}, 2\right),\left(-\frac{3 n-4}{2}, 2\right),\left(\frac{3 n+8}{2}, 2\right),\left(-\frac{3 n-2}{2}, 2\right),\left(\frac{3 n}{2}, 2\right)\right) \text {; } \\
& T_{8}=\left(\left(-\frac{3 n+2}{2}, 2\right),\left(\frac{3 n+14}{2}, 2\right),\left(-\frac{3 n+4}{2}, 2\right),\left(\frac{3 n+4}{2}, 2\right),\left(-\frac{3 n+6}{2}, 2\right),\left(\frac{3 n+6}{2}, 2\right), \ldots, \frac{\left(-\left(\frac{3 n+2}{2}+3 i\right), 2\right),\left(\frac{3 n+14}{2}+3 i, 2\right),}{}\right.
\end{aligned}
$$

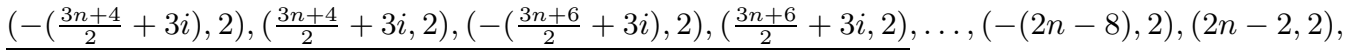

$$
\begin{aligned}
& (-(2 n-7), 2),(2 n-7,2),(-(2 n-6), 2),(2 n-6,2)), 0 \leq i \leq \frac{n-18}{6} \text {; } \\
& T_{9}=((-(2 n-5), 2),(2 n, 2),(-(2 n-2), 2),(2 n-4,2),(-(2 n-4), 2),(2 n-3,2),(-(2 n-1), 2)) .
\end{aligned}
$$

Case 2.2: $k \equiv 9(\bmod 24)$ and $k \geq 57$.

$$
\begin{aligned}
T_{2}= & \left((-(n+2), 1),(n+3,1),(-(n+3), 1),(n+1,1),(-(n+1), 1),(n+2,1), \ldots, \frac{(-(n+2+3 i), 1),(n+3+3 i, 1),}{}\right. \\
& \frac{(-(n+3+3 i), 1),(n+1+3 i, 1),(-(n+1+3 i), 1),(n+2+3 i, 1), \ldots,\left(-\frac{3 n-4}{2}, 1\right),\left(\frac{3 n-2}{2}, 1\right),}{\left.\left(-\frac{3 n-2}{2}, 1\right),\left(\frac{3 n-6}{2}, 1\right),\left(-\frac{3 n-6}{2}, 1\right),\left(\frac{3 n-4}{2}, 1\right)\right), 0 \leq i \leq \frac{n-8}{6} ;} \\
T_{3}= & \left(\left(-\frac{3 n+2}{2}, 1\right),\left(-\frac{3 n}{2}, 1\right),\left(\frac{3 n+4}{2}, 1\right)\right) ;
\end{aligned}
$$




$$
\begin{aligned}
T_{4}= & \left(\left(\frac{3 n}{2}, 1\right),\left(-\frac{3 n+8}{2}, 1\right),\left(\frac{3 n+2}{2}, 1\right),\left(-\frac{3 n+4}{2}, 1\right),\left(\frac{3 n+10}{2}, 1\right),\left(-\frac{3 n+6}{2}, 1\right), \ldots, \frac{\left(\frac{3 n}{2}+3 i, 1\right),\left(-\left(\frac{3 n+8}{2}+3 i\right), 1\right),}{}\right. \\
& \frac{\left(\frac{3 n+2}{2}+3 i, 1\right),\left(-\left(\frac{3 n+4}{2}+3 i\right), 1\right),\left(\frac{3 n+10}{2}+3 i, 1\right),\left(-\left(\frac{3 n+6}{2}+3 i\right), 1\right), \ldots,(2 n-7,1),(-(2 n-3), 1),}{(2 n-6,1),(-(2 n-5), 1),(2 n-2,1),(-(2 n-4), 1)), 0 \leq i \leq \frac{n-14}{6} ;} \\
T_{5}= & ((2 n-4,1),(-2 n, 1),(2 n-3,1),(-(2 n-2), 1),(2 n, 1),(2 n, 3),(-2 n, 2),(n, 2),(n+3,2),(-(n+2), 2), \\
& (n+2,2)) ; \\
T_{6}= & \left((-(n+1), 2),(n+5,2),(-(n+5), 2),(n+6,2),(-(n+3), 2),(n+4,2), \ldots, \frac{(-(n+1+3 i), 2),(n+5+3 i, 2),}{}\right. \\
& \frac{(-(n+5+3 i), 2),(n+6+3 i, 2),(-(n+3)+3 i), 2),(n+4+3 i, 2), \ldots,\left(-\frac{3 n-12}{2}, 2\right),\left(\frac{3 n-4}{2}, 2\right),}{\left.\left(-\frac{3 n-4}{2}, 2\right),\left(\frac{3 n-2}{2}, 2\right),\left(-\frac{3 n-8}{2}, 2\right),\left(\frac{3 n-6}{2}, 2\right)\right), 0 \leq i \leq \frac{n-14}{6} ;} \\
T_{7}= & \left(\left(-\frac{3 n-6}{2}, 2\right),\left(\frac{3 n+4}{2}, 2\right),\left(-\frac{n}{2}, 2\right),\left(\frac{3 n}{2}, 2\right),\left(-\frac{3 n+2}{2}, 2\right)\right) ; \\
T_{8}= & \left(\left(\frac{3 n+2}{2}, 2\right),\left(-\frac{3 n+8}{2}, 2\right),\left(\frac{3 n+6}{2}, 2\right),\left(-\frac{3 n+6}{2}, 2\right),\left(\frac{3 n+10}{2}, 2\right),\left(-\frac{3 n-2}{2}, 2\right), \ldots,\left(\frac{3 n+2}{2}+3 i, 2\right),\left(-\left(\frac{3 n+8}{2}+3 i\right), 2\right),\right. \\
& \frac{\left(\frac{3 n+6}{2}+3 i, 2\right),\left(-\left(\frac{3 n+6}{2}+3 i\right), 2\right),\left(\frac{3 n+10}{2}+3 i, 2\right),\left(-\left(\frac{3 n-2}{2}+3 i\right), 2\right), \ldots,(2 n-9,2),(-(2 n-6), 2),}{(2 n-7,2),(-(2 n-7), 2),(2 n-5,2),(-(2 n-11), 2)), 0 \leq i \leq \frac{n-20}{6} ;} \\
T_{9}= & ((2 n-6,2),(-(2 n-4), 2),(2 n-3,2),(-(2 n-8), 2),(2 n, 2),(-(2 n-5), 2),(2 n-4,2),(-(2 n-2), 2), \\
& (2 n-2,2),(-(2 n-1), 2)) .
\end{aligned}
$$

Case 2.3: $k \equiv 17(\bmod 24)$ and $k \geq 65$.

$T_{2}=((-(n+2), 1),(n+3,1),(-(n+3), 1),(n+1,1),(-(n+1), 1),(n+2,1), \ldots,(-(n+2+3 i), 1),(n+3+3 i, 1)$, $\underline{(-(n+3+3 i), 1),(n+1+3 i, 1),(-(n+1+3 i), 1),(n+2+3 i, 1)}, \ldots,\left(-\frac{3 n-6}{2}, 1\right),\left(\frac{3 n-4}{2}, 1\right)$, $\left.\left(-\frac{3 n-4}{2}, 1\right),\left(\frac{3 n-8}{2}, 1\right),\left(-\frac{3 n-8}{2}, 1\right),\left(\frac{3 n-6}{2}, 1\right)\right), 0 \leq i \leq \frac{n-10}{6} ;$

$T_{3}=\left(\left(-\frac{3 n}{2}, 1\right),\left(\frac{3 n-2}{2}, 1\right),\left(-\frac{3 n-2}{2}, 1\right),\left(\frac{3 n+6}{2}, 1\right),\left(\frac{3 n}{2}, 1\right)\right) ;$

$T_{4}=\left(\left(\frac{3 n+2}{2}, 1\right),\left(-\frac{3 n+6}{2}, 1\right),\left(\frac{3 n+4}{2}, 1\right),\left(-\frac{3 n+2}{2}, 1\right),\left(\frac{3 n+12}{2}, 1\right),\left(-\frac{3 n+4}{2}, 1\right), \ldots,\left(\frac{3 n+2}{2}+3 i, 1\right),\left(-\left(\frac{3 n+6}{2}+3 i\right), 1\right)\right.$,

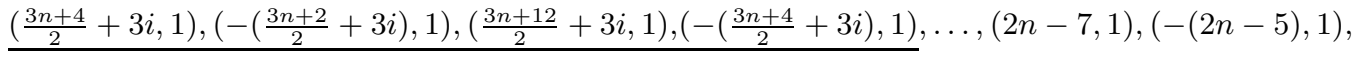
$(2 n-6,1),(-(2 n-7), 1),(2 n-2,1),(-(2 n-6), 1)), 0 \leq i \leq \frac{n-16}{6} ;$

$T_{5}=((2 n-4,1),(-(2 n-3), 1),(2 n-3,1),(-(2 n-2), 1),(-2 n, 1),(-(2 n-4), 1),(2 n, 1),(2 n, 3),(-2 n, 2),(n, 2)$, $(n+3,2),(-(n+3), 2),(n+4,2),(-(n+1), 2),(n+2,2))$;

$T_{6}=((-(n+2), 2),(n+6,2),(-(n+6), 2),(n+7,2),(-(n+4), 2),(n+5,2), \ldots, \underline{(-(n+2+3 i), 2),(n+6+3 i, 2),}$

$\underline{(-(n+6+3 i), 2),(n+7+3 i, 2),(-(n+4+3 i), 2),(n+5+3 i, 2)}, \ldots,\left(-\frac{3 n-12}{2}, 2\right),\left(\frac{3 n-4}{2}, 2\right)$, $\left.\left(-\frac{3 n-4}{2}, 2\right),\left(\frac{3 n-2}{2}, 2\right),\left(-\frac{3 n-8}{2}, 2\right),\left(\frac{3 n-6}{2}, 2\right)\right), 0 \leq i \leq \frac{n-16}{6}$;

$T_{7}=\left(\left(-\frac{3 n-6}{2}, 2\right),\left(\frac{3 n+4}{2}, 2\right),\left(-\frac{n}{2}, 2\right),\left(\frac{3 n}{2}, 2\right),\left(-\frac{3 n+2}{2}, 2\right)\right)$;

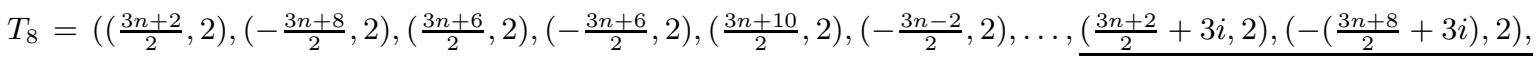
$\left(\frac{3 n+6}{2}+3 i, 2\right),\left(-\left(\frac{3 n+6}{2}+3 i\right), 2\right),\left(\frac{3 n+10}{2}+3 i, 2\right),\left(-\left(\frac{3 n-2}{2}+3 i\right), 2\right), \ldots,(2 n-10,2),(-(2 n-7), 2)$, $(2 n-8,2),(-(2 n-8), 2),(2 n-6,2),(-(2 n-12), 2)), 0 \leq i \leq \frac{n-22}{6} ;$

$T_{9}=((2 n-7,2),(-(2 n-2), 2),(2 n-5,2),(-(2 n-6), 2),(2 n-4,2),(-(2 n-9), 2),(2 n-3,2),(-(2 n-5), 2)$,

$(2 n, 2),(-(2 n-4), 2),(2 n-2,2),(-(2 n-1), 2))$.

\section{$4 \quad k$-ARCS $(4 k+1)$ for $k \equiv 3(\bmod 4)$}

In this section, we will prove the existence of a $k$-ARCS $(4 k+1)$ for $k \equiv 3(\bmod 4)$. 
Lemma 4.1. For any $k \geq 11$ and $k \equiv 3(\bmod 4)$, there exists a $k$-ARCS $(4 k+1)$.

Proof: Let $v=4 k+1$ and $k=4 n+3, n \geq 2$. We use Lemma 2.1 to construct a $k$-ARCS $(v)$ with $V\left(K_{v}\right)=\left(\mathbb{Z}_{k} \times \mathbb{Z}_{4}\right) \cup\{\infty\}$. Three of the required parameters are $\left(a_{1}, b_{1}\right)=(0,3)$ and $d_{3}=\frac{k-1}{2}$. The other required parameters $a_{2}, b_{2}, d_{2}$ and 8 cycles in $F_{1}=\left\{C_{1}, C_{2}, C_{3}, C_{4}\right\}$ and $F_{2}=\left\{C_{5}, C_{6}, C_{7}, C_{8}\right\}$ are listed as below.

The cycle $C_{1}$ is the concatenation of the sequences $T_{1},(0,0)$, and $T_{2}$, where

$T_{1}=((n, 0),(-n, 1), \ldots, \underline{(n-i, 0),(-(n-i), 1)}, \ldots,(1,0),(-1,1)), 0 \leq i \leq n-1 ;$

$T_{2}=((1,1),(-1,0), \ldots,(1+i, 1),(-(1+i), 0), \ldots,(n+1,1),(-(n+1), 0)), 0 \leq i \leq n$.

The cycle $C_{2}$ is the concatenation of the sequences $T_{1}, T_{2}, T_{3}$, and $(0,2)$, where

$T_{1}=((1,2),(-1,3), \ldots, \underline{(1+i, 2),(-(1+i), 3)}, \ldots,(n, 2),(-n, 3)), 0 \leq i \leq n-1 ;$

$T_{2}=((n+1,2),(n+1,3))$;

$T_{3}=((-n, 2),(n, 3), \ldots, \underline{(-(n-i), 2),(n-i, 3)}, \ldots,(-1,2),(1,3)), 0 \leq i \leq n-1$.

The cycle $C_{3}$ is the concatenation of the sequences $T_{1}, T_{2}$, and $T_{3}$, where

$T_{1}=((n+2,1),(-(n+2), 2), \ldots, \underline{(n+2+i, 1),(-(n+2+i), 2)}, \ldots,(2 n, 1),(-2 n, 2)), 0 \leq i \leq n-2 ;$

$T_{2}=((-(2 n+1), 1),(2 n+1,2), \ldots, \underline{(-(2 n+1-i), 1),(2 n+1-i, 2)}, \ldots,(-(n+2), 1),(n+2,2)), 0 \leq i \leq n-1$;

$\left.T_{3}=((-(n+1), 1),(-(n+1), 2)),(-(2 n+1), 0),(-(2 n+1), 2),(0,1)\right)$.

The cycle $C_{4}$ is the concatenation of the sequences $\infty, T_{1}, T_{2}, T_{3}$, and $T_{4}$, where

$T_{1}=((-(2 n+1), 3),(2 n+1,3), \ldots, \underline{(-(2 n+1-i), 3),(2 n+1-i, 3)}, \ldots,(-(n+2), 3),(n+2,3)), 0 \leq i \leq n-1 ;$

$T_{2}=((-(n+1), 3),(n+1,0))$;

$T_{3}=((n+2,0),(-(n+2), 0), \ldots, \underline{(n+2+i, 0),(-(n+2+i), 0)}, \ldots,(2 n, 0),(-2 n, 0)), 0 \leq i \leq n-2 ;$

$T_{4}=((2 n+1,0),(2 n+1,1))$.

For $k=11$, the other four cycles are listed as below.

$k=11:\left(a_{2}, b_{2}\right)=\left(\frac{k+1}{2}, 2\right), d_{2}=2$.

$C_{5}=((0,1),(0,3),(1,1),(2,3),(4,1),(1,3),(5,1),(-4,3),(2,1),(5,3),(-1,1))$;

$C_{6}=((0,0),(3,3),(1,0),(-2,3),(3,0),(4,3),(4,0),(-3,3),(-1,0),(-5,3),(-4,0))$;

$C_{7}=((2,2),(-3,1),(1,2),(-5,0),(4,2),(-3,0),(5,2),(2,0),(3,2),(-2,0),(-3,2))$;

$C_{8}=(\infty,(5,0),(-2,2),(-4,1),(-2,1),(3,1),(-5,1),(-1,3),(0,2),(-4,2),(-1,2))$.

For $k \geq 15$, the two cycles $C_{5}$ and $C_{6}$ are defined as below.

The cycle $C_{5}$ is the concatenation of the sequences $T_{1}, T_{2},(0,1), T_{3}$, and $T_{4}$, where

$T_{1}=((n+2,1),(-(n+1), 3))$;

$T_{2}=((n, 1),(-n, 3), \ldots, \underline{(n-i, 1),(-(n-i), 3)}, \ldots,(1,1),(-1,3)), 0 \leq i \leq n-1$;

$T_{3}=((1,3),(-1,1), \ldots, \underline{(1+i, 3),(-(1+i), 1)}, \ldots,(n-1,3),(-(n-1), 1)), 0 \leq i \leq n-2 ;$

$T_{4}=((n, 3),(-(n+1), 1))$.

The cycle $C_{6}$ is the concatenation of the sequences $T_{1}, T_{2}, T_{3}$, and $(n+1,0)$, where

$T_{1}=((-(n+2), 0),(n+2,3), \ldots, \underline{(-(n+2+i), 0),(n+2+i, 3)}, \ldots,(-(2 n+1), 0),(2 n+1,3)), 0 \leq i \leq n-1 ;$

$T_{2}=((2 n+1,0),(0,3),(-1,0),(-(2 n+1), 3))$;

$T_{3}=((2 n, 0),(-2 n, 3), \ldots, \underline{(2 n-i, 0),(-(2 n-i), 3)}, \ldots,(n+2,0),(-(n+2), 3)), 0 \leq i \leq n-2$. 
For the last two cycles $C_{7}$ and $C_{8}$, when $k=15,19,23,27,35$, they are listed as below. $k=15:\left(a_{2}, b_{2}\right)=\left(\frac{3 k-5}{4}, 2\right), d_{2}=4$.

$C_{7}=((0,2),(-7,1),(-1,2),(0,0),(1,2),(3,0),(7,2),(1,0),(-2,2),(-4,0),(3,2),(-2,0),(6,2),(-3,0),(-7,2))$;

$C_{8}=(\infty,(2,0),(-3,2),(-5,1),(6,1),(7,1),(-6,1),(-3,1),(4,1),(4,3),(5,2),(2,2),(-4,2),(-6,2),(4,2))$.

$k=19:\left(a_{2}, b_{2}\right)=\left(\frac{k+1}{2}, 2\right), d_{2}=2$.

$C_{7}=((0,2),(-9,1),(-7,2),(0,0),(1,2),(2,0),(4,2),(1,0),(7,2),(-4,0),(-8,2),(3,0),(-2,2),(4,0),(-6,2)$,

$(-3,0),(2,2),(-5,0),(5,2))$;

$C_{8}=(\infty,(-2,0),(-4,2),(7,1),(-8,1),(9,1),(-7,1),(-6,1),(8,1),(-4,1),(5,1),(5,3),(6,2),(3,2),(9,2)$,

$(-3,2),(8,2),(-1,2),(-5,2))$.

$k=23:\left(a_{2}, b_{2}\right)=\left(\frac{3 k-1}{4}, 2\right), d_{2}=2$.

$C_{7}=((1,2),(-9,1),(2,2),(-2,0),(4,2),(-3,0),(5,2),(-4,0),(6,2),(-5,0),(8,2),(5,0),(-4,2),(4,0)$,

$(3,2),(1,0),(-1,2),(3,0),(-3,2),(0,0),(-5,2),(-6,0),(10,2))$;

$C_{8}=(\infty,(2,0),(-9,2),(-11,1),(8,1),(-7,1),(11,1),(10,1),(-10,1),(-8,1),(9,1),(-5,1),(6,1),(6,3)$,

$(7,2),(0,2),(-11,2),(-8,2),(9,2),(-10,2),(-2,2),(11,2),(-7,2))$.

$k=27:\left(a_{2}, b_{2}\right)=\left(\frac{k+1}{2}, 2\right), d_{2}=2$.

$C_{7}=((0,2),(-13,1),(-11,2),(0,0),(1,2),(2,0),(4,2),(1,0),(5,2),(-7,0),(2,2),(4,0),(9,2),(-6,0),(7,2)$,

$(-4,0),(3,2),(-5,0),(13,2),(3,0),(-5,2),(-2,0),(-8,2),(-3,0),(-7,2),(6,0),(-4,2))$;

$C_{8}=(\infty,(5,0),(-2,2),(13,1),(11,1),(-12,1),(-11,1),(-8,1),(9,1),(-9,1),(12,1),(-10,1),(10,1),(-6,1)$,

$(7,1),(7,3),(8,2),(11,2),(-6,2),(6,2),(12,2),(-10,2),(10,2),(-3,2),(-12,2),(-1,2),(-9,2))$.

$k=35:\left(a_{2}, b_{2}\right)=\left(\frac{k+1}{2}, 2\right), d_{2}=2$.

$C_{7}=((1,2),(-15,1),(2,2),(-2,0),(3,2),(-3,0),(4,2),(-5,0),(5,2),(-6,0),(6,2),(-7,0),(7,2),(-8,0)$,

$(8,2),(-9,0),(11,2),(8,0),(9,2),(7,0),(-9,2),(5,0),(-8,2),(4,0),(-7,2),(3,0),(-6,2),(-4,0)$,

$(-5,2),(0,0),(-4,2),(2,0),(-1,2),(6,0),(-2,2))$;

$C_{8}=(\infty,(1,0),(-16,2),(17,1),(-16,1),(16,1),(-12,1),(-17,1),(-13,1),(13,1),(-14,1),(15,1),(14,1)$,

$(-10,1),(11,1),(-11,1),(12,1),(-8,1),(9,1),(9,3),(10,2),(-12,2),(12,2),(-11,2),(14,2),(-13,2)$,

$(16,2),(-10,2),(-15,2),(13,2),(-3,2),(15,2),(0,2),(-14,2),(17,2))$.

For the last two cycles $C_{7}$ and $C_{8}$ of other values, we distinguish the following 2 cases.

Case 1: $k \equiv 3(\bmod 8)$ and $k \geq 43$. Here, $\left(a_{2}, b_{2}\right)=\left(\frac{k+1}{2}, 2\right), d_{2}=2$.

The cycle $C_{7}$ is the concatenation of the sequences $S_{1}, S_{2}, \ldots, S_{5},\left(-\frac{n}{2}, 0\right), S_{6}$, and $S_{7}$, where

$S_{1}=((1,2),(-(2 n-1), 1))$;

$S_{2}=\left((2,2),(-2,0), \ldots, \underline{(2+i, 2),(-(2+i), 0)}, \ldots,\left(\frac{n-2}{2}, 2\right),\left(-\frac{n-2}{2}, 0\right)\right), 0 \leq i \leq \frac{n-6}{2} ;$

$S_{3}=\left(\left(\frac{n}{2}, 2\right),\left(-\frac{n+2}{2}, 0\right), \ldots, \underline{\left(\frac{n}{2}+i, 2\right),\left(-\left(\frac{n+2}{2}+i\right), 0\right)}, \ldots,(n, 2),(-(n+1), 0)\right), 0 \leq i \leq \frac{n}{2}$;

$S_{4}=((n+3,2),(n, 0),(n+1,2),(n-1,0),(-(n+1), 2)) ;$

$S_{5}=\left((n-3,0),(-n, 2), \ldots, \underline{(n-3-i, 0),(-(n-i), 2)}, \ldots,\left(\frac{n-2}{2}, 0\right),\left(-\frac{n+4}{2}, 2\right)\right), 0 \leq i \leq \frac{n-4}{2}$.

For the sequences $S_{6}, S_{7}$, and the cycle $C_{8}$, we distinguish 3 subcases.

Case 1.1: $k \equiv 3(\bmod 24)$ and $k \geq 51$. 


$$
\begin{aligned}
S_{6}= & \left(\left(-\frac{n+2}{2}, 2\right),\left(\frac{n-8}{2}, 0\right),\left(-\frac{n}{2}, 2\right),\left(\frac{n-4}{2}, 0\right),\left(-\frac{n-8}{2}, 2\right),\left(\frac{n-6}{2}, 0\right), \ldots, \frac{\left(-\left(\frac{n+2}{2}-3 i\right), 2\right),\left(\frac{n-8}{2}-3 i, 0\right),\left(-\left(\frac{n}{2}-3 i\right),\right.}{}\right. \\
& \frac{\left.2),\left(\frac{n-4}{2}-3 i, 0\right),\left(-\left(\frac{n-8}{2}-3 i\right), 2\right),\left(\frac{n-6}{2}-3 i, 0\right), \ldots,(-10,2),(5,0),(-9,2),(7,0),(-5,2),(6,0)\right),}{0 \leq i \leq \frac{n-18}{6}} \\
S_{7}= & ((-7,2),(3,0),(-6,2),(2,0),(-4,2),(0,0),(-3,2),(4,0),(-1,2),(n-2,0),(-2,2)) .
\end{aligned}
$$

The cycle $C_{8}$ is the concatenation of the sequences $\infty, T_{1}, T_{2}, \ldots, T_{9}$, where

$$
\begin{aligned}
& T_{1}=((1,0),(-2 n, 2),(2 n+1,1),(-2 n, 1),(-(2 n+1), 1),(-(2 n-3), 1),(2 n-1,1),(-(2 n-2), 1),(2 n, 1)) ; \\
& T_{2}=((-(2 n-5), 1),(2 n-2,1),(-(2 n-4), 1),(2 n-4,1),(-(2 n-6), 1),(2 n-3,1), \ldots, \underline{(-(2 n-5-3 i), 1),} \\
& \underline{(2 n-2-3 i, 1),(-(2 n-4-3 i), 1),(2 n-4-3 i, 1),(-(2 n-6-3 i), 1),(2 n-3-3 i, 1)}, \ldots,\left(-\frac{3 n+2}{2}, 1\right), \\
& \left.\left(\frac{3 n+8}{2}, 1\right),\left(-\frac{3 n+4}{2}, 1\right),\left(\frac{3 n+4}{2}, 1\right),\left(-\frac{3 n}{2}, 1\right),\left(\frac{3 n+6}{2}, 1\right)\right), 0 \leq i \leq \frac{n-12}{6} \text {; } \\
& T_{3}=\left(\left(\frac{3 n}{2}, 1\right),\left(-\frac{3 n-2}{2}, 1\right),\left(\frac{3 n+2}{2}, 1\right)\right) \text {; } \\
& T_{4}=\left(\left(-\frac{3 n-6}{2}, 1\right),\left(\frac{3 n-2}{2}, 1\right),\left(-\frac{3 n-4}{2}, 1\right),\left(\frac{3 n-6}{2}, 1\right),\left(-\frac{3 n-8}{2}, 1\right),\left(\frac{3 n-4}{2}, 1\right), \ldots,\left(-\left(\frac{3 n-6}{2}-3 i\right), 1\right),\left(\frac{3 n-2}{2}-3 i, 1\right),\right. \\
& \frac{\left(-\left(\frac{3 n-4}{2}-3 i\right), 1\right),\left(\frac{3 n-6}{2}-3 i, 1\right),\left(-\left(\frac{3 n-8}{2}-3 i\right), 1\right),\left(\frac{3 n-4}{2}-3 i, 1\right)}{(-(n+4), 1),(n+3,1),(-(n+2), 1),(n+4,1)), 0 \leq i \leq \frac{n-12}{6} ;},(-(n+3), 1),(n+5,1), \\
& T_{5}=((-n, 1),(n+1,1),(n+1,3)) \text {; } \\
& T_{6}=((n+2,2),(-(n+4), 2),(n+4,2),(-(n+3), 2),(n+6,2),(-(n+5), 2), \ldots, \underline{(n+2+3 i, 2),(-(n+4+3 i), 2),} \\
& \underline{(n+4+3 i, 2),(-(n+3+3 i), 2),(n+6+3 i, 2),(-(n+5+3 i), 2)}, \ldots,\left(\frac{3 n-8}{2}, 2\right),\left(-\frac{3 n-4}{2}, 2\right),\left(\frac{3 n-4}{2}, 2\right), \\
& \left.\left(-\frac{3 n-6}{2}, 2\right),\left(\frac{3 n}{2}, 2\right),\left(-\frac{3 n-2}{2}, 2\right)\right), 0 \leq i \leq \frac{n-12}{6} \text {; } \\
& T_{7}=\left(\left(\frac{3 n-2}{2}, 2\right),\left(-\frac{3 n+2}{2}, 2\right),\left(\frac{3 n+2}{2}, 2\right),\left(-\frac{3 n}{2}, 2\right),\left(\frac{3 n+8}{2}, 2\right),\left(-\frac{n-2}{2}, 2\right)\right) \text {; }
\end{aligned}
$$

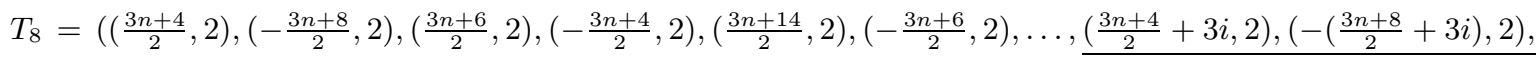

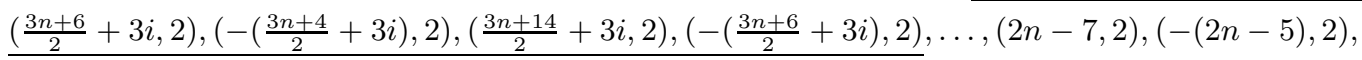

$$
\begin{aligned}
& (2 n-6,2),(-(2 n-7), 2),(2 n-2,2),(-(2 n-6), 2)), 0 \leq i \leq \frac{n-18}{6} \text {; } \\
& T_{9}=((2 n-4,2),(-(2 n-3), 2),(2 n-3,2),(-(2 n-2), 2),(0,2),(2 n-1,2),(-(2 n-1), 2),(2 n, 2),(-(2 n-4), \\
& 2),(2 n+1,2),(-(n+2), 2)) \text {. }
\end{aligned}
$$

Case 1.2: $k \equiv 11(\bmod 24)$ and $k \geq 59$.

$S_{6}=\left(\left(-\frac{n+2}{2}, 2\right),\left(\frac{n-8}{2}, 0\right),\left(-\frac{n}{2}, 2\right),\left(\frac{n-4}{2}, 0\right),\left(-\frac{n-8}{2}, 2\right),\left(\frac{n-6}{2}, 0\right), \ldots, \underline{\left(-\left(\frac{n+2}{2}-3 i\right), 2\right),\left(\frac{n-8}{2}-3 i, 0\right),\left(-\left(\frac{n}{2}-3 i\right),\right.}\right.$ $\left.2),\left(\frac{n-4}{2}-3 i, 0\right),\left(-\left(\frac{n-8}{2}-3 i\right), 2\right),\left(\frac{n-6}{2}-3 i, 0\right), \ldots,(-8,2),(3,0),(-7,2),(5,0),(-3,2),(4,0)\right)$, $0 \leq i \leq \frac{n-14}{6}$ $S_{7}=((-5,2),(0,0),(-4,2),(2,0),(-1,2),(n-2,0),(-2,2))$.

The cycle $C_{8}$ is the concatenation of the sequences $\infty, T_{1}, T_{2}, \ldots, T_{9}$, where

$$
\begin{aligned}
T_{1}= & ((1,0),(-2 n, 2),(2 n+1,1),(-2 n, 1),(2 n, 1),(-(2 n-4), 1),(-(2 n+1), 1),(-(2 n-3), 1),(2 n-3,1), \\
& (-(2 n-2), 1),(2 n-1,1)) ; \\
T_{2}= & ((-(2 n-7), 1),(2 n-2,1),(-(2 n-5), 1),(2 n-6,1),(-(2 n-6), 1),(2 n-4,1), \ldots,(-(2 n-7-3 i), 1), \\
& \frac{(2 n-2-3 i, 1),(-(2 n-5-3 i), 1),(2 n-6-3 i, 1),(-(2 n-6-3 i), 1),(2 n-4-3 i, 1), \ldots,\left(-\frac{3 n}{2}, 1\right),}{\left.\left(\frac{3 n+10}{2}, 1\right),\left(-\frac{3 n+4}{2}, 1\right),\left(\frac{3 n+2}{2}, 1\right),\left(-\frac{3 n+2}{2}, 1\right),\left(\frac{3 n+6}{2}, 1\right)\right), 0 \leq i \leq \frac{n-14}{6} ;} \\
T_{3}= & \left(\left(\frac{3 n+4}{2}, 1\right),\left(-\frac{3 n-4}{2}, 1\right)\right) ; \\
T_{4}= & \left(\left(\frac{3 n}{2}, 1\right),\left(-\frac{3 n-2}{2}, 1\right),\left(\frac{3 n-4}{2}, 1\right),\left(-\frac{3 n-6}{2}, 1\right),\left(\frac{3 n-2}{2}, 1\right),\left(-\frac{3 n-10}{2}, 1\right), \ldots, \frac{\left(\frac{3 n}{2}-3 i, 1\right),\left(-\left(\frac{3 n-2}{2}-3 i\right), 1\right),}{}\right. \\
& \frac{\left(\frac{3 n-4}{2}-3 i, 1\right),\left(-\left(\frac{3 n-6}{2}-3 i\right), 1\right),\left(\frac{3 n-2}{2}-3 i, 1\right),\left(-\left(\frac{3 n-10}{2}-3 i\right), 1\right), \ldots,(n+7,1),(-(n+6), 1),}{}
\end{aligned}
$$




$$
\begin{aligned}
& (n+5,1),(-(n+4), 1),(n+6,1),(-(n+2), 1)), 0 \leq i \leq \frac{n-14}{6} ; \\
T_{5}= & ((n+3,1),(-(n+3), 1),(n+4,1),(-n, 1),(n+1,1),(n+1,3)) ; \\
T_{6}= & \left((n+2,2),(-(n+4), 2),(n+4,2),(-(n+3), 2),(n+6,2),(-(n+5), 2), \ldots, \frac{(n+2+3 i, 2),(-(n+4+3 i), 2),}{}\right. \\
& \quad \frac{(n+4+3 i, 2),(-(n+3+3 i), 2),(n+6+3 i, 2),(-(n+5+3 i), 2), \ldots,\left(\frac{3 n-4}{2}, 2\right),\left(-\frac{3 n}{2}, 2\right),\left(\frac{3 n}{2}, 2\right),}{\left.\left(-\frac{3 n-2}{2}, 2\right),\left(\frac{3 n+4}{2}, 2\right),\left(-\frac{3 n+2}{2}, 2\right)\right), 0 \leq i \leq \frac{n-8}{6} ;} \\
T_{7}= & \left(\left(\frac{3 n+2}{2}, 2\right),\left(-\frac{3 n+6}{2}, 2\right),\left(\frac{3 n+6}{2}, 2\right),\left(-\frac{3 n+4}{2}, 2\right),\left(\frac{3 n+10}{2}, 2\right),\left(-\frac{n-2}{2}, 2\right)\right) ; \\
T_{8}= & \left(\left(\frac{3 n+12}{2}, 2\right),\left(-\frac{3 n+10}{2}, 2\right),\left(\frac{3 n+8}{2}, 2\right),\left(-\frac{3 n+12}{2}, 2\right),\left(\frac{3 n+16}{2}, 2\right),\left(-\frac{3 n+8}{2}, 2\right), \ldots,\left(\frac{3 n+12}{2}+3 i, 2\right),\left(-\left(\frac{3 n+10}{2}+3 i\right),\right.\right. \\
& \frac{2),\left(\frac{3 n+8}{2}+3 i, 2\right),\left(-\left(\frac{3 n+12}{2}+3 i\right), 2\right),\left(\frac{3 n+16}{2}+3 i, 2\right),\left(-\left(\frac{3 n+8}{2}+3 i\right), 2\right), \ldots,(2 n-4,2),(-(2 n-5), 2),}{(2 n-6,2),(-(2 n-4), 2),(2 n-2,2),(-(2 n-6), 2)), 0 \leq i \leq \frac{n-20}{6} ;} \\
T_{9}= & ((2 n-1,2),(-(2 n-2), 2),(2 n-3,2),(-(2 n-1), 2),(2 n, 2),(0,2),(2 n+1,2),(-(2 n-3), 2),(-(n+2), 2)) .
\end{aligned}
$$

Case 1.3: $k \equiv 19(\bmod 24)$ and $k \geq 43$.

$$
\begin{aligned}
S_{6}= & \left(\left(-\frac{n+2}{2}, 2\right),\left(\frac{n-8}{2}, 0\right),\left(-\frac{n}{2}, 2\right),\left(\frac{n-4}{2}, 0\right),\left(-\frac{n-8}{2}, 2\right),\left(\frac{n-6}{2}, 0\right), \ldots, \frac{\left(-\left(\frac{n+2}{2}-3 i\right), 2\right),\left(\frac{n-8}{2}-3 i, 0\right),\left(-\left(\frac{n}{2}-3 i\right),\right.}{}\right. \\
& \frac{\left.2),\left(\frac{n-4}{2}-3 i, 0\right),\left(-\left(\frac{n-8}{2}-3 i\right), 2\right),\left(\frac{n-6}{2}-3 i, 0\right), \ldots,(-9,2),(4,0),(-8,2),(6,0),(-4,2),(5,0)\right),}{} \quad \\
S_{7} \leq i \leq \frac{n-16}{6} ; & ((-6,2),(0,0),(-3,2),(2,0),(-5,2),(3,0),(-1,2),(n-2,0),(-2,2)) .
\end{aligned}
$$

The cycle $C_{8}$ is the concatenation of the sequences $\infty, T_{1}, T_{2}, \ldots, T_{9}$, where

$$
\begin{aligned}
& T_{1}=((1,0),(-2 n, 2),(2 n+1,1),(2 n-1,1),(-2 n, 1),(-(2 n+1), 1)) \text {; } \\
& T_{2}=((2 n-4,1),(-(2 n-3), 1),(2 n-2,1),(-(2 n-4), 1),(2 n, 1),(-(2 n-2), 1), \ldots, \underline{(2 n-4-3 i, 1)} \text {, } \\
& (-(2 n-3-3 i), 1),(2 n-2-3 i, 1),(-(2 n-4-3 i), 1),(2 n-3 i, 1),(-(2 n-2-3 i), 1), \ldots, \\
& \left.\left(\frac{3 n+2}{2}, 1\right),\left(-\frac{3 n+4}{2}, 1\right),\left(\frac{3 n+6}{2}, 1\right),\left(-\frac{3 n+2}{2}, 1\right),\left(\frac{3 n+10}{2}, 1\right),\left(-\frac{3 n+6}{2}, 1\right)\right), 0 \leq i \leq \frac{n-10}{6} \text {; } \\
& T_{3}=\left(\left(-\frac{3 n}{2}, 1\right),\left(\frac{3 n+4}{2}, 1\right)\right) \text {; }
\end{aligned}
$$

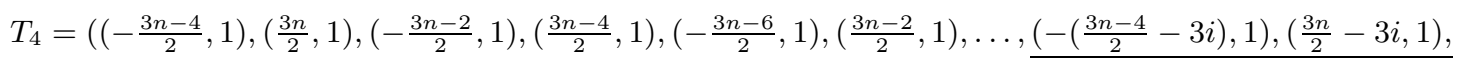

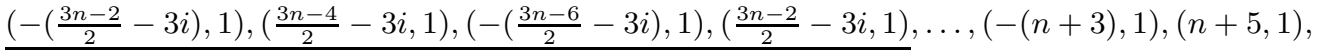

$$
\begin{aligned}
& (-(n+4), 1),(n+3,1),(-(n+2), 1),(n+4,1)), 0 \leq i \leq \frac{n-10}{6} \\
& T_{5}=((-n, 1),(n+1,1),(n+1,3)) \text {; } \\
& T_{6}=((n+2,2),(-(n+4), 2),(n+4,2),(-(n+3), 2),(n+6,2),(-(n+5), 2), \ldots,(n+2+3 i, 2),(-(n+4+3 i), 2) \text {, } \\
& \underline{(n+4+3 i, 2),(-(n+3+3 i), 2),(n+6+3 i, 2),(-(n+5+3 i), 2)}, \ldots,\left(\frac{3 n-6}{2}, 2\right),\left(-\frac{3 n-2}{2}, 2\right),\left(\frac{3 n-2}{2}, 2\right), \\
& \left.\left(-\frac{3 n-4}{2}, 2\right),\left(\frac{3 n+2}{2}, 2\right),\left(-\frac{3 n}{2}, 2\right)\right), 0 \leq i \leq \frac{n-10}{6} \text {; } \\
& T_{7}=\left(\left(\frac{3 n}{2}, 2\right),\left(-\frac{n-2}{2}, 2\right)\right)
\end{aligned}
$$

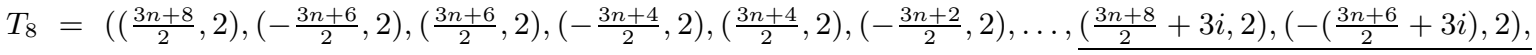

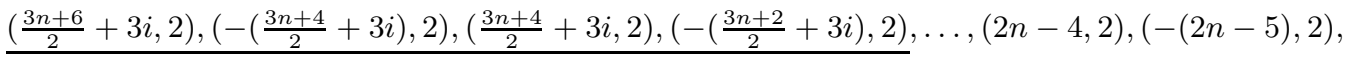

$$
\begin{aligned}
& (2 n-5,2),(-(2 n-6), 2),(2 n-6,2),(-(2 n-7), 2)), 0 \leq i \leq \frac{n-16}{6} \text {; } \\
& T_{9}=((2 n-1,2),(-(2 n-2), 2),(0,2),(2 n+1,2),(-(2 n-3), 2),(2 n-2,2),(-(2 n-4), 2),(2 n-3,2),(-(2 n-1) \text {, } \\
& 2),(2 n, 2),(-(n+2), 2)) \text {. }
\end{aligned}
$$

Case 2: $k \equiv 7(\bmod 8)$ and $k \geq 31$.

In this case the cycle $C_{7}$ is the concatenation of the sequences $S_{1}, S_{2}, S_{3},(n+3,2), S_{4}$, $S_{5}, S_{6}$, and $S_{7}$, where 


$$
\begin{aligned}
& S_{1}=((1,2),(-(2 n-1), 1)) ; \\
& S_{2}=\left((2,2),(-2,0), \ldots,(2+i, 2),(-(2+i), 0), \ldots,\left(\frac{n-1}{2}, 2\right),\left(-\frac{n-1}{2}, 0\right)\right), 0 \leq i \leq \frac{n-5}{2} ; \\
& S_{3}=\left(\left(\frac{n+3}{2}, 2\right),\left(-\frac{n+1}{2}, 0\right), \ldots, \frac{\left.\left(\frac{n+3}{2}+i, 2\right),\left(-\left(\frac{n+1}{2}+i\right), 0\right), \ldots,(n+1,2),(-n, 0)\right), 0 \leq i \leq \frac{n-1}{2} ;}{S_{4}}=\left((n, 0),(-(n-1), 2), \ldots, \underline{(n-i, 0),(-(n-1-i), 2)}, \ldots,\left(\frac{n+5}{2}, 0\right),\left(-\frac{n+3}{2}, 2\right)\right), 0 \leq i \leq \frac{n-5}{2} ;\right. \\
& S_{5}=\left(\left(\frac{n+3}{2}, 0\right),\left(\frac{n+1}{2}, 2\right)\right) .
\end{aligned}
$$

For the sequences $S_{6}, S_{7}$, and the cycle $C_{8}$, we distinguish 3 subcases.

Case 2.1: $k \equiv 7(\bmod 24)$ and $k \geq 31$. Here, $\left(a_{2}, b_{2}\right)=\left(\frac{3 k-5}{4}, 2\right), d_{2}=4$.

$$
\begin{aligned}
S_{6}= & \left(\left(\frac{n-3}{2}, 0\right),\left(-\frac{n-5}{2}, 2\right),\left(\frac{n-1}{2}, 0\right),\left(-\frac{n-3}{2}, 2\right),\left(\frac{n+1}{2}, 0\right),\left(-\frac{n-1}{2}, 2\right), \ldots, \frac{\left(\frac{n-3}{2}-3 i, 0\right),\left(-\left(\frac{n-5}{2}-3 i\right), 2\right),}{}\right. \\
& \frac{\left(\frac{n-1}{2}-3 i, 0\right),\left(-\left(\frac{n-3}{2}-3 i\right), 2\right),\left(\frac{n+1}{2}-3 i, 0\right),\left(-\left(\frac{n-1}{2}-3 i\right), 2\right), \ldots,(5,0),(-4,2),(6,0),(-5,2),}{(7,0),(-6,2)), 0 \leq i \leq \frac{n-13}{6} ;} \\
S_{7}= & ((2,0),(0,2),(3,0),(-2,2),(4,0),(-3,2),(1,0),(-n, 2),(-(n+1), 0),(2 n, 2)) .
\end{aligned}
$$

For $k=31,55$, the cycle $C_{8}$ is listed as below.

$k=31$ :

$$
\begin{aligned}
C_{8}= & (\infty,(0,0),(-15,2),(14,1),(12,1),(-14,1),(-15,1),(-12,1),(15,1),(-10,1),(13,1),(-11,1),(10,1), \\
& (-9,1),(11,1),(-7,1),(8,1),(8,3),(9,2),(11,2),(-11,2),(15,2),(-1,2),(-13,2),(-10,2),(13,2), \\
& (-4,2),(-14,2),(-8,2),(12,2),(-12,2)) . \\
k=55: & \\
C_{8}= & (\infty,(0,0),(-27,2),(26,1),(-26,1),(23,1),(-27,1),(27,1),(-24,1),(22,1),(-23,1),(25,1),(-22,1), \\
& (-20,1),(24,1),(-19,1),(19,1),(-18,1),(21,1),(-21,1),(20,1),(-16,1),(16,1),(-15,1),(18,1), \\
& (-17,1),(17,1),(-13,1),(14,1),(14,3),(15,2),(-18,2),(17,2),(-17,2),(19,2),(-19,2),(18,2), \\
& (-21,2),(20,2),(-20,2),(22,2),(-22,2),(21,2),(-25,2),(27,2),(-1,2),(25,2),(-7,2),(24,2), \\
& (-26,2),(23,2),(-24,2),(-14,2),(-16,2),(-23,2)) .
\end{aligned}
$$

For any $k \geq 79$, the cycle $C_{8}$ is the concatenation of the sequences $\infty, T_{1}, T_{2}, \ldots, T_{9}$, where

$$
\begin{aligned}
& T_{1}=((0,0),(-(2 n+1), 2),(2 n, 1),(-2 n, 1),(2 n-3,1)(-(2 n+1), 1),(2 n+1,1)(-(2 n-2), 1)) ; \\
& T_{2}=((2 n-6,1),(-(2 n-3), 1),(2 n-1,1),(-(2 n-4), 1),(2 n-2,1),(-(2 n-5), 1), \ldots, \underline{(2 n-6-3 i, 1)}, \\
& \underline{(-(2 n-3-3 i), 1),(2 n-1-3 i, 1),(-(2 n-4-3 i), 1),(2 n-2-3 i, 1),(-(2 n-5-3 i), 1), \ldots,}, \\
& \left.\left(\frac{3 n+7}{2}, 1\right),\left(-\frac{3 n+13}{2}, 1\right),\left(\frac{3 n+17}{2}, 1\right),\left(-\frac{3 n+11}{2}, 1\right),\left(\frac{3 n+15}{2}, 1\right),\left(-\frac{3 n+9}{2}, 1\right)\right), 0 \leq i \leq \frac{n-19}{6} \text {; } \\
& T_{3}=\left(\left(\frac{3 n+5}{2}, 1\right),\left(-\frac{3 n+7}{2}, 1\right),\left(\frac{3 n+11}{2}, 1\right),\left(-\frac{3 n+5}{2}, 1\right),\left(-\frac{3 n+1}{2}, 1\right),\left(\frac{3 n+9}{2}, 1\right),\left(-\frac{3 n-1}{2}, 1\right),\left(\frac{3 n-1}{2}, 1\right),\left(-\frac{3 n-3}{2}, 1\right),\right. \\
& \left.\left(\frac{3 n+3}{2}, 1\right),\left(-\frac{3 n+3}{2}, 1\right),\left(\frac{3 n+1}{2}, 1\right),\left(-\frac{3 n-7}{2}, 1\right)\right) \text {; }
\end{aligned}
$$

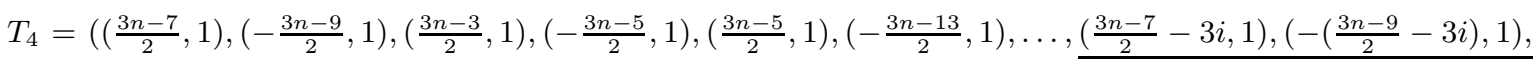

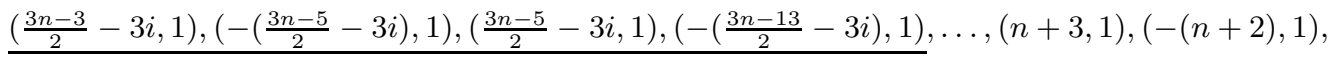

$$
\begin{aligned}
& (n+5,1),(-(n+4), 1),(n+4,1),(-n, 1)), 0 \leq i \leq \frac{n-13}{6} \text {; } \\
& T_{5}=((n+1,1),(n+1,3)) \text {; } \\
& T_{6}=((n+2,2),(-(n+5), 2),(n+4,2),(-(n+4), 2),(n+6,2),(-(n+6), 2), \ldots, \underline{(n+2+3 i, 2),(-(n+5+3 i), 2),} \\
& \underline{(n+4+3 i, 2),(-(n+4+3 i), 2),(n+6+3 i, 2),(-(n+6+3 i), 2)}, \ldots,\left(\frac{3 n-3}{2}, 2\right),\left(-\frac{3 n+3}{2}, 2\right),\left(\frac{3 n+1}{2}, 2\right), \\
& \left.\left(-\frac{3 n+1}{2}, 2\right),\left(\frac{3 n+5}{2}, 2\right),\left(-\frac{3 n+5}{2}, 2\right)\right), 0 \leq i \leq \frac{n-7}{6} \text {; }
\end{aligned}
$$


$T_{7}=\left(\left(\frac{3 n+3}{2}, 2\right),\left(\frac{3 n+13}{2}, 2\right),\left(-\frac{3 n+9}{2}, 2\right),\left(\frac{3 n+7}{2}, 2\right),\left(-\frac{3 n+11}{2}, 2\right),\left(\frac{3 n+9}{2}, 2\right),\left(-\frac{n+1}{2}, 2\right)\right)$;

$T_{8}=\left(\left(\frac{3 n+11}{2}, 2\right),\left(-\frac{3 n+17}{2}, 2\right),\left(\frac{3 n+15}{2}, 2\right),\left(-\frac{3 n+15}{2}, 2\right),\left(\frac{3 n+19}{2}, 2\right),\left(-\frac{3 n+7}{2}, 2\right), \ldots,\left(\frac{3 n+11}{2}+3 i, 2\right),\left(-\left(\frac{3 n+17}{2}+3 i\right)\right.\right.$, $2),\left(\frac{3 n+15}{2}+3 i, 2\right),\left(-\left(\frac{3 n+15}{2}+3 i\right), 2\right),\left(\frac{3 n+19}{2}+3 i, 2\right),\left(-\left(\frac{3 n+7}{2}+3 i\right), 2\right), \ldots,(2 n-7,2),(-(2 n-4), 2)$,

$(2 n-5,2),(-(2 n-5), 2),(2 n-3,2),(-(2 n-9), 2)), 0 \leq i \leq \frac{n-25}{6}$;

$T_{9}=((2 n-4,2),(-(2 n-2), 2),(2 n-2,2),(-(2 n-3), 2),(-(n+1), 2),(-(n+3), 2),(-2 n, 2),(-(2 n-6), 2)$,

$(2 n-1,2),(-1,2),(2 n+1,2),(-(2 n-1), 2))$.

Case 2.2: $k \equiv 15(\bmod 24)$ and $k \geq 39$. Here, $\left(a_{2}, b_{2}\right)=\left(\frac{3 k-5}{4}, 2\right), d_{2}=4$.

$S_{6}=\left(\left(\frac{n-3}{2}, 0\right),\left(-\frac{n-5}{2}, 2\right),\left(\frac{n-1}{2}, 0\right),\left(-\frac{n-3}{2}, 2\right),\left(\frac{n+1}{2}, 0\right),\left(-\frac{n-1}{2}, 2\right), \ldots, \frac{\left(\frac{n-3}{2}-3 i, 0\right),\left(-\left(\frac{n-5}{2}-3 i\right), 2\right),}{2}\right.$

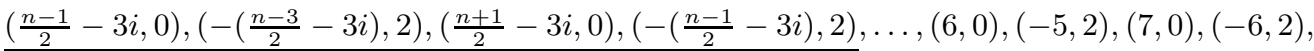

$(8,0),(-7,2)), 0 \leq i \leq \frac{n-15}{6}$;

$S_{7}=((3,0),(-3,2),(4,0),(-4,2),(5,0),(0,2),(2,0),(-2,2),(1,0),(-n, 2),(-(n+1), 0),(2 n, 2))$.

For $k=39$, , the cycle $C_{8}$ is listed as below.

$C_{8}=(\infty,(0,0),(-19,2),(18,1),(-18,1),(15,1),(-19,1),(19,1),(-16,1),(-14,1),(16,1),(-15,1),(17,1)$,

$(-12,1),(12,1),(-11,1),(14,1),(-13,1),(13,1),(-9,1),(10,1),(10,3),(11,2),(-14,2),(13,2)$,

$(-13,2),(15,2),(-15,2),(14,2),(-5,2),(19,2),(16,2),(-16,2),(17,2),(-1,2),(-17,2),(-12,2)$,

$(-10,2),(-18,2))$.

For any $k \geq 63$, the cycle $C_{8}$ is the concatenation of the sequences $\infty, T_{1}, T_{2}, \ldots, T_{9}$, where

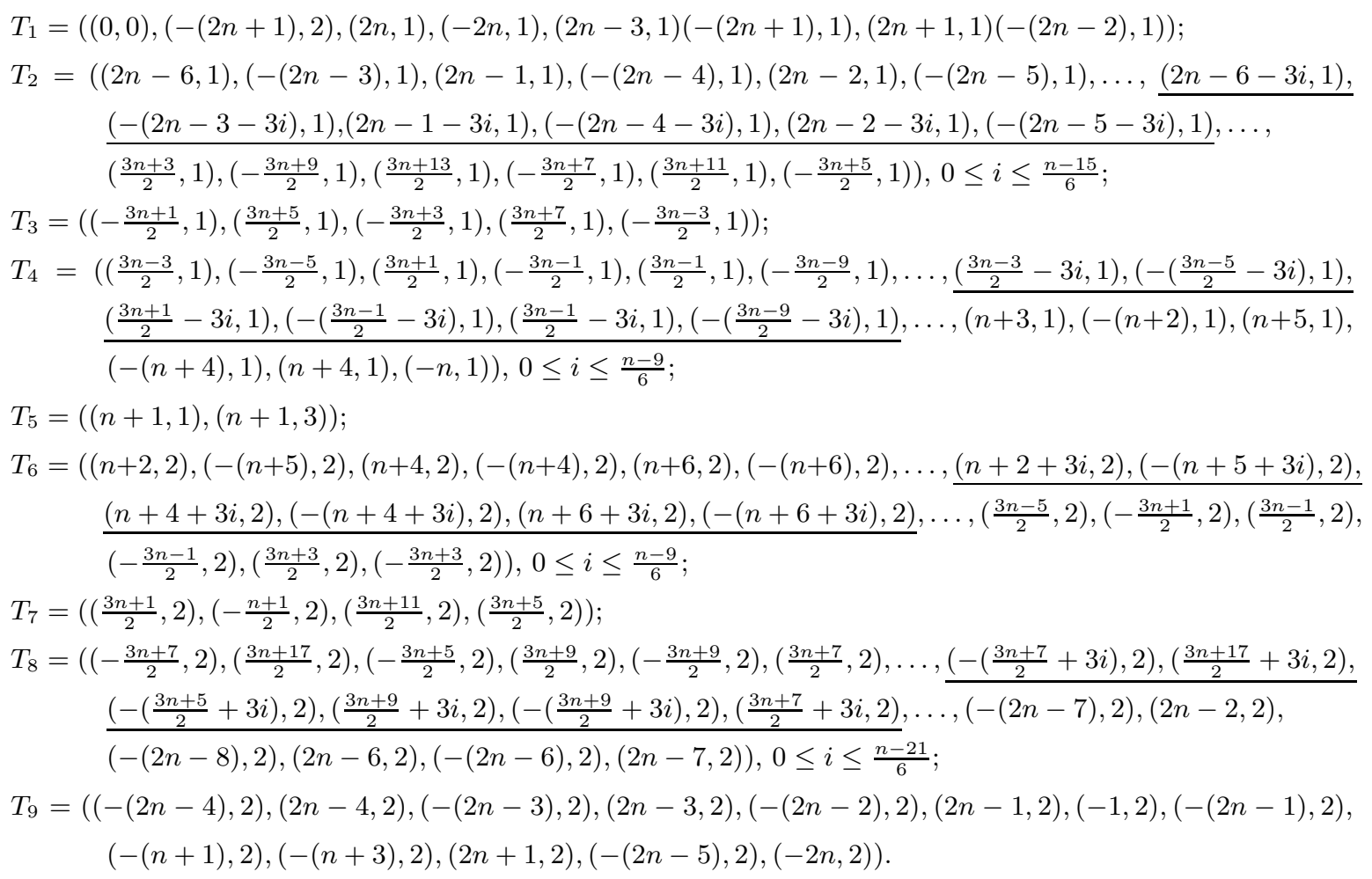

Case 2.3: $k \equiv 23(\bmod 24)$ and $k \geq 47$. Here, $\left(a_{2}, b_{2}\right)=\left(\frac{3 k-1}{4}, 2\right), d_{2}=2$. 


$$
\begin{aligned}
S_{6}= & \left(\left(\frac{n-3}{2}, 0\right),\left(-\frac{n-5}{2}, 2\right),\left(\frac{n-1}{2}, 0\right),\left(-\frac{n-3}{2}, 2\right),\left(\frac{n+1}{2}, 0\right),\left(-\frac{n-1}{2}, 2\right), \ldots,\left(\frac{n-3}{2}-3 i, 0\right),\left(-\left(\frac{n-5}{2}-3 i\right), 2\right),\right. \\
& \frac{\left(\frac{n-1}{2}-3 i, 0\right),\left(-\left(\frac{n-3}{2}-3 i\right), 2\right),\left(\frac{n+1}{2}-3 i, 0\right),\left(-\left(\frac{n-1}{2}-3 i\right), 2\right), \ldots,(7,0),(-6,2),(8,0),(-7,2),(9,0),}{(-8,2)), 0 \leq i \leq \frac{n-17}{6} ;} \\
S_{7}= & ((4,0),(-3,2),(3,0),(-5,2),(6,0),(-4,2),(5,0),(0,2),(2,0),(-2,2),(1,0),(-n, 2),(-(n+1), 0),(2 n, 2)) .
\end{aligned}
$$

For $k=47$, the cycle $C_{8}$ is listed as below.

$$
\begin{aligned}
C_{8}= & (\infty,(0,0),(-23,2),(22,1),(-22,1),(19,1),(-23,1),(23,1),(-20,1),(20,1),(-19,1),(-17,1),(21,1), \\
& (-16,1),(17,1),(-18,1),(18,1),(-14,1),(14,1),(-13,1),(16,1),(-15,1),(15,1),(-11,1),(12,1), \\
& (12,3),(13,2),(-16,2),(18,2),(-15,2),(17,2),(-14,2),(16,2),(19,2),(-6,2),(21,2),(-21,2),(15,2), \\
& (-20,2),(-1,2),(23,2),(-17,2),(20,2),(-19,2),(-13,2),(-22,2),(-18,2)) .
\end{aligned}
$$

For any $k \geq 71$, the cycle $C_{8}$ is the concatenation of the sequences $\infty, T_{1}, T_{2}, \ldots, T_{9}$, where

$$
\begin{aligned}
& T_{1}=((0,0),(-(2 n+1), 2),(2 n, 1),(-2 n, 1),(2 n-3,1)(-(2 n+1), 1),(2 n+1,1)(-(2 n-2), 1)) ; \\
& T_{2}=((2 n-6,1),(-(2 n-3), 1),(2 n-1,1),(-(2 n-4), 1),(2 n-2,1),(-(2 n-5), 1), \ldots, \underline{(2 n-6-3 i, 1)} \text {, } \\
& \underline{(-(2 n-3-3 i), 1),(2 n-1-3 i, 1),(-(2 n-4-3 i), 1),(2 n-2-3 i, 1),(-(2 n-5-3 i), 1)}, \ldots, \\
& \left.\left(\frac{3 n+5}{2}, 1\right),\left(-\frac{3 n+11}{2}, 1\right),\left(\frac{3 n+15}{2}, 1\right),\left(-\frac{3 n+9}{2}, 1\right),\left(\frac{3 n+13}{2}, 1\right),\left(-\frac{3 n+7}{2}, 1\right)\right), 0 \leq i \leq \frac{n-17}{6} ; \\
& T_{3}=\left(\left(\frac{3 n+7}{2}, 1\right),\left(-\frac{3 n+5}{2}, 1\right),\left(-\frac{3 n+1}{2}, 1\right),\left(\frac{3 n+9}{2}, 1\right),\left(-\frac{3 n-1}{2}, 1\right),\left(\frac{3 n+1}{2}, 1\right),\left(-\frac{3 n+3}{2}, 1\right),\left(\frac{3 n+3}{2}, 1\right),\left(-\frac{3 n-5}{2}, 1\right)\right) ;
\end{aligned}
$$

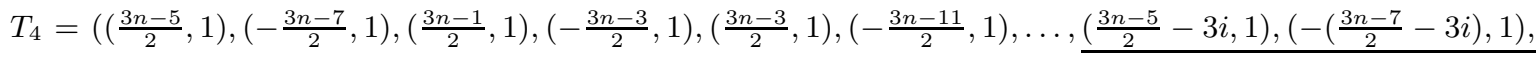

$$
\begin{aligned}
& \left(\frac{3 n-1}{2}-3 i, 1\right),\left(-\left(\frac{3 n-3}{2}-3 i\right), 1\right),\left(\frac{3 n-3}{2}-3 i, 1\right),\left(-\left(\frac{3 n-11}{2}-3 i\right), 1\right), \ldots,(n+3,1),(-(n+2), 1), \\
& (n+5,1),(-(n+4), 1),(n+4,1),(-n, 1)), 0 \leq i \leq \frac{n-11}{6} \text {; } \\
& T_{5}=((n+1,1),(n+1,3)) \text {; } \\
& T_{6}=((n+2,2),(-(n+5), 2),(n+7,2),(-(n+4), 2),(n+6,2),(-(n+3), 2), \ldots, \underline{(n+2+3 i, 2),(-(n+5+3 i), 2),} \\
& \underline{(n+7+3 i, 2),(-(n+4+3 i), 2),(n+6+3 i, 2),(-(n+3+3 i), 2)}, \ldots,\left(\frac{3 n-7}{2}, 2\right),\left(-\frac{3 n-1}{2}, 2\right),\left(\frac{3 n+3}{2}, 2\right), \\
& \left.\left(-\frac{3 n-3}{2}, 2\right),\left(\frac{3 n+1}{2}, 2\right),\left(-\frac{3 n-5}{2}, 2\right)\right), 0 \leq i \leq \frac{n-11}{6} \text {; } \\
& T_{7}=\left(\left(\frac{3 n-1}{2}, 2\right),\left(\frac{3 n+5}{2}, 2\right),\left(-\frac{n+1}{2}, 2\right),\left(-\frac{3 n+1}{2}, 2\right),\left(\frac{3 n+7}{2}, 2\right),\left(-\frac{3 n+5}{2}, 2\right),\left(\frac{3 n+11}{2}, 2\right),\left(-\frac{3 n+3}{2}, 2\right)\right) ;
\end{aligned}
$$

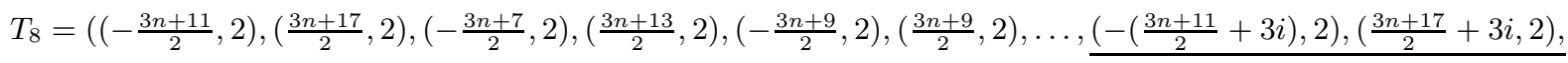

$$
\begin{aligned}
& \left(-\left(\frac{3 n+7}{2}+3 i\right), 2\right),\left(\frac{3 n+13}{2}+3 i, 2\right),\left(-\left(\frac{3 n+9}{2}+3 i\right), 2\right),\left(\frac{3 n+9}{2}+3 i, 2\right), \ldots,(-(2 n-6), 2),(2 n-3,2), \\
& (-(2 n-8), 2),(2 n-5,2),(-(2 n-7), 2),(2 n-7,2)), 0 \leq i \leq \frac{n-23}{6} \text {; } \\
& T_{9}=((-(2 n-3), 2),(2 n-2,2),(-(2 n-5), 2),(2 n-1,2),(-(2 n-1), 2),(-1,2),(2 n+1,2),(-(2 n-4), 2) \text {, } \\
& (2 n-4,2),(-2 n, 2),(-(n+2), 2),(n+4,2),(-(2 n-2), 2)) \text {. }
\end{aligned}
$$

\section{Concluding remarks}

Combining Theorems 1.1, 1.2, and Lemmas 3.1, 4.1, we have proved Theorem 1.3. This completes the proof of the existence of almost resolvable cycle systems with odd cycle length. For the even case, Lemma 2.1 is still useful for certain subcases.

We are working on the case $k=4 m+2$. But it should be mentioned that Lemma 2.1 can 
not be applied to solve the existence of a $k$-almost resolvable cycle system when $k=2^{m}$. So there is still a long way to go before the whole problem can be solved completely.

As an application, almost resolvable cycle systems can be used to construct some solutions to the Hamilton-Waterloo problem [30]. The Hamilton-Waterloo problem $\operatorname{HWP}(v ; m, n ; \alpha, \beta)$ is the problem of determining whether the complete graph $K_{v}$ (for $v$ odd) or $K_{v}$ minus a 1-factor $I$ (for $v$ even) has a 2-factorization in which there are exactly $\alpha C_{m}$-factors and $\beta C_{n}$-factors. We denote by $\operatorname{HWP}(v ; m, n)$ the set of $(\alpha, \beta)$ for which a solution to $\operatorname{HWP}(v ; m, n ; \alpha, \beta)$ exists. For recent results on the Hamilton-Waterloo problem, we refer the reader to [5, 7-11, 16, 17, 21, 23, 28, 29]. As a by-product, the following theorem can be obtained by combining Theorem 1.3 of this paper, and Theorems 1.4 and 3.5, Constructions 3.11 and 3.13 in [30].

Theorem 5.1. If $k \geq 3$ is odd and $t \geq 1$, then $(\alpha, \beta) \in \operatorname{HWP}(k(2 k t+1) ; k, 2 k t+1)$ if and only if $\alpha, \beta \geq 0$ and $\alpha+\beta=\frac{k(2 k t+1)-1}{2}$, except possibly when:

1. $t=1$.

(1) $k=5: \beta \in\{1,2,3\}$;

(2) $k=7: \beta \in\{1,2,3,5\}$;

(3) $k \geq 9: \beta \in\{1,2,3,5,7\}$.

2. $t=2$.

(1) $k=3: \beta \in[1,2 k-1] \cup\{2 k+1,2 k+3\}$;

(2) $k=5,7,9: \beta \in\{1,2,3,5,7\}$.

3. $t \geq 3$.

(1) $k=3$ :

- $t$ is odd : $\beta \in\{1,3,5, \ldots, 3 t-4,3 t-2\} \cup\{2,9 t-3,9 t-1\}$

- $t$ is even : $\beta \in\{1,3,5, \ldots, 3 t+1,3 t+3\} \cup\{2,9 t-3,9 t-1\}$;

(2) $k \geq 5: \beta \in\{1,2,3,5,7\}$.

\section{Acknowledgments}

We would like to thank the anonymous referees for their careful read and helpful comments and suggestions which greatly improved the quality of this paper.

\section{References}

[1] P. Adams, E. J. Billington, D. G. Hoffman, C. C. Lindner, The generalized almost resolvable cycle system problem, Combinatorica 30 (2010), 617-625.

[2] B. Alspach, H. Gavlas, Cycle decompositions of $K_{n}$ and $K_{n}-I$, J. Combin. Theory Ser. B 81 (2001), 77-99.

[3] B. Alspach, R. Haggkvist, Some observations on the Oberwolfach problem, Journal of Graph Theory 9 (1985), 177-187. 
[4] B. Alspach, P. J. Schellenberg, D. R. Stinson, D. Wagner, The Oberwolfach problem and factors of uniform odd length cycles, J. Combin. Theory Ser. A 52 (1989), 20-43.

[5] J. Asplund, D. Kamin, M. Keranen, A. Pastine, S. Özkan, On the Hamilton-Waterloo problem with triangle factors and $C_{3 x}$-factors, Australas. J. Combin. 64 (2016), 458-474.

[6] E. J. Billington, D. G. Hoffman, C. C. Lindner, M. Meszka, Almost resolvable minimum coverings of complete graphs with 4-cycles, Australas. J. Combin. 50 (2011), 73-85.

[7] S. Bonvicini, M. Buratti, Octahedral, dicyclic and special linear solutions of some unsolved Hamilton-Waterloo problems, Ars Math. Contemp. 14 (2018), 1-14.

[8] M. Buratti, P. Danziger, A cyclic solution for an infinite class of Hamilton-Waterloo problems, Graphs and Combin. 32 (2016), 521-531.

[9] A. Burgess, P. Danziger, T. Traetta, On the Hamilton-Waterloo problem with odd orders, J. Combin. Des. 25 (2017), 258-287.

[10] A. Burgess, P. Danziger, T. Traetta, On the Hamilton-Waterloo problem with odd cycle lengths, preprint.

[11] A. Burgess, P. Danziger, T. Traetta, On the Hamilton-Waterloo problem with cycle lengths of distinct parities, preprint.

[12] H. Cao, M. Niu, C. Tang, On the existence of cycle frames and almost resolvable cycle systems, Discrete Math. 311 (2011), 2220-2232.

[13] I. J. Dejter, C. C. Lindner, M. Meszka, C. A. Rodger, Corrigendum/addendum to: almost resolvable 4-cycle systems, J. Combin. Math. Combin. Comput. 66 (2008), 297298.

[14] I. J. Dejter, C. C. Lindner, C. A. Rodger, M. Meszka, Almost resolvable 4-cycle systems, J. Combin. Math. Combin. Comput. 63 (2007), 173-181.

[15] D. G. Hoffman, P. J. Schellenberg, The existence of $C_{k}$-factorizations of $K_{2 n}-F$, Discrete Math. 97 (1991), 243-250.

[16] M. Keranen, A. Pastine, A generalization of the Hamilton-Waterloo problem on complete equipartite graphs, J. Combin. Des. 25 (2017), 431-468.

[17] H. Lei, H. Fu, The Hamilton-Waterloo problem for triangle-factors and heptagon-factors, Graphs Combin. 32 (2016), 271-278.

[18] C. C. Lindner, M. Meszka, A. Rosa, Almost resolvable cycle systems - an analogue of Hanani triple systems, J. Combin. Des. 17 (2009), 404-410.

[19] J. Liu, A generalization of the Oberwolfach problem and $C_{t}$-factorizations of complete equipartite graphs, J. Combin. Des. 8 (2000), 42-49.

[20] J. Liu, The equipartite Oberwolfach problem with uniform tables, J. Combin. Theory Ser. A 101 (2003), 20-34.

[21] F. Merola, T. Traetta, Infinitely many cyclic solutions to the Hamilton-Waterloo problem with odd length cycles, Discrete Math. 339 (2016), 2267-2283.

[22] M. Niu, H. Cao, More results on cycle frames and almost resolvable cycle systems, Discrete Math. 312 (2012), 3392-3405. 
[23] U. Odabaşı, S. Özkan, The Hamilton-Waterloo problem with $C_{4}$ and $C_{m}$ factors, Discrete Math. 339 (2016), 263-269.

[24] W. L. Piotrowski, The solution of the bipartite analogue of the Oberwolfach problem, Discrete Math. 97 (1991), 339-356.

[25] R. Rees, Two new direct product-type constructions for resolvable group-divisible designs, J. Combin. Des. 1 (1993), 15-26.

[26] M. Šajna, Cycle decompositions: complete graphs and fixed length cycles, J. Combin. Des. 10 (2002), 27-78.

[27] S. A. Vanstone, D. R. Stinson, P. J. Schellenberg, A. Rosa, R. Rees, C. J. Colbourn, M. W. Carter, J. E. Carter, Hanani triple systems, Israel J. Math. 83 (1993), 305-319.

[28] L. Wang, H. Cao, A note on the Hamilton-Waterloo problem with $C_{8}$-factors and $C_{m^{-}}$ factors, Discrete Math. (2017), http://dx.doi.org/10.1016/j.disc.2017.06.024.

[29] L. Wang, F. Chen, H. Cao, The Hamilton-Waterloo problem for $C_{3}$-factors and $C_{n^{-}}$ factors, J. Combin. Des. 25 (2017), 385-418.

[30] L. Wang, S. Lu, H. Cao, Further results on almost resolvable cycle systems and the Hamilton-Waterloo problem, J. Combin. Des. (2017), DOI: 10.1002/jcd.21571. 